\title{
EXTREMAL PROBLEMS FOR POLYNOMIALS WITH EXPONENTIAL WEIGHTS
}

\author{
BY \\ H. N. MHASKAR ${ }^{1}$ AND E. B. SAFF $^{2}$
}

\begin{abstract}
Dedicated to Professor J. L. Ullman on the occasion of his 60 th birthday, in recognition of his contributions to the study of orthogonal polynomials and his motivating influence on the present work.
\end{abstract}

ABSTRACT. For the extremal problem:

$$
E_{n, r}(\alpha):=\min \left\|\exp \left(-|x|^{\alpha}\right)\left(x^{n}+\cdots\right)\right\|_{L^{r}}, \quad \alpha>0,
$$

where $L^{r}(0<r \leqslant \infty)$ denotes the usual integral norm over $\mathbf{R}$, and the minimum is taken over all monic polynomials of degree $n$, we describe the asymptotic form of the error $E_{n, r}(\alpha)($ as $n \rightarrow \infty)$ as well as the limiting distribution of the zeros of the corresponding extremal polynomials. The case $r=2$ yields new information regarding the polynomials $\left\{p_{n}(\alpha ; x)=\gamma_{n}(\alpha) x^{n}+\cdots\right\}$ which are orthonormal on $\mathbf{R}$ with respect to $\exp \left(-2|x|^{\alpha}\right)$. In particular, it is shown that a conjecture of Freud concerning the leading coefficients $\gamma_{n}(\alpha)$ is true in a Cesàro sense. Furthermore a contracted zero distribution theorem is proved which, unlike a previous result of Ullman, does not require the truth of the Freud's conjecture. For $r=\infty, \alpha>0$ we also prove that, if $\operatorname{deg} P_{n}(x) \leqslant n$, the norm $\left\|\exp \left(-|x|^{\alpha}\right) P_{n}(x)\right\|_{L^{\infty}}$ is attained on the finite interval

$$
\left[-\left(n / \lambda_{\alpha}\right)^{1 / \alpha},\left(n / \lambda_{\alpha}\right)^{1 / \alpha}\right], \text { where } \lambda_{\alpha}=\Gamma(\alpha) / 2^{\alpha-2}\{\Gamma(\alpha / 2)\}^{2} .
$$

Extensions of Nikolskii-type inequalities are also given.

1. Introduction. In this paper, we shall investigate a class of extremal problems arising in the theory of weighted polynomial approximation on the whole real line. We shall concentrate on the weight functions $\exp \left(-|x|^{\alpha}\right), \alpha>0$, although some of our methods apply to a more general class of weight functions.

Let $\mathscr{P}_{n}$ denote the collection of algebraic polynomials of degree at most $n$. For $\alpha>0, r>0$, put

$$
E_{n, r}(\alpha):=\inf _{q_{n-1} \in \mathscr{P}_{n-1}}\left(\int_{-\infty}^{\infty} \exp \left(-r|x|^{\alpha}\right)\left|x^{n}-q_{n-1}(x)\right|^{r} d x\right)^{1 / r}
$$

where, if $r=\infty$, the supremum norm is meant. Our purpose is to study the behavior of $E_{n, r}(\alpha)$ as well as that of the polynomial $T_{n, r}(\alpha ; x)=x^{n}+\cdots \in \mathscr{P}_{n}$ for which

Received by the editors January 2, 1983.

1980 Mathematics Subject Classification. Primary 41A17; Secondary 33A65.

Key words and phrases. Orthogonal polynomials, exponential weights, error estimates, zero distribution, Nikolskii inequalities.

'Part of this author's work was conducted at the University of Michigan, Ann Arbor.

${ }^{2}$ Research supported in part by the National Science Foundation. 
$E_{n, r}(\alpha)$ is attained. Specifically, we shall determine:

(a) the limiting behavior of $E_{n, r}(\alpha)$ as $n \rightarrow \infty$;

(b) the limiting distribution of the zeros of the extremal polynomials $T_{n, r}(\alpha ; x)$ as $n \rightarrow \infty$;

(c) the asymptotic behavior of $\left|T_{n, r}(\alpha ; z)\right|$, as $n$ and $z$ tend to infinity, $z \in \mathbf{C}$.

The cases $r=\infty$ and $r=2$ are of particular interest. The case $r=\infty$ is analogous to the study of the class of incomplete polynomials introduced by Lorentz [15] in the sense that our weight function $\exp \left(-|x|^{\alpha}\right)$ vanishes at the endpoints $( \pm \infty)$ of the interval. When the weight is $\exp (-x)$ and the interval in question is $[0, \infty)$, the corresponding sup norm extremal problem was investigated in detail by Saff and Varga [29]. Several of their results can be obtained from the theorems of the present paper by means of elementary transformations.

The study of the case $r=2$ is really that of the polynomials

$$
p_{n}(\alpha ; x)=\gamma_{n}(\alpha) x^{n}+\cdots \in \mathscr{P}_{n}, \quad \gamma_{n}(\alpha)>0(n=1,2, \ldots),
$$

which are normal and orthogonal on the real line with respect to the weight $\exp \left(-2|x|^{\alpha}\right), \alpha>0$. It is known [4] that (apart from the multiplicative factor $\gamma_{n}(\alpha)$ ) these are precisely the extremal polynomials $T_{n, 2}(\alpha ; x)$, that $E_{n, 2}(\alpha)$ is the reciprocal of the leading coefficient $\gamma_{n}(\alpha)$ of $p_{n}(\alpha ; x)$ and that the sequence $\left\{p_{n}(\alpha ; x)\right\}$ satisfies the recurrence relations

$$
x p_{n}(x)=\frac{\gamma_{n}}{\gamma_{n+1}} p_{n+1}(x)+\frac{\gamma_{n-1}}{\gamma_{n}} p_{n-1}(x) \quad\left(\gamma_{j}=\gamma_{j}(\alpha), p_{j}(x)=p_{j}(\alpha ; x)\right)
$$

(We caution the reader that our notation differs slightly from the standard one used in the literature on weighted approximation, where $p_{n}(\alpha ; x)$ is usually denoted by $p_{n}\left(w_{\alpha}^{2} ; x\right)$.)

Various quantities associated with these orthonormal polynomials $\left\{p_{n}\right\}$ were investigated by Freud, Nevai, Ullman and others, especially for the case $\alpha \geqslant 1$. For example, Nevai [21] proved that if $\alpha \geqslant 1$, then $\gamma_{n-1} / \gamma_{n}$ and the largest zero $X_{n}$ of $p_{n}$ are both of the same order of magnitude, namely $n^{1 / \alpha}$. Freud, in his investigations [7, 8] of more general weighted polynomial approximation, obtained analogous results concerning the order of magnitude of $X_{n}$ and

$$
\Gamma_{n}:=\max _{1 \leqslant k \leqslant n} \frac{\gamma_{k-1}}{\gamma_{k}}
$$

He also proved in [9] that if $\alpha$ is a positive even integer, then

$$
\begin{aligned}
2^{-\alpha+1}(2 \alpha)^{-1 / \alpha} & \leqslant \liminf _{n \rightarrow \infty} n^{-1 / \alpha} \frac{\gamma_{n-1}}{\gamma_{n}} \leqslant \frac{1}{2}\left(\frac{1}{\lambda_{\alpha}}\right)^{1 / \alpha}, \\
\frac{1}{2}\left(\frac{1}{\lambda_{\alpha}}\right)^{1 / \alpha} & \leqslant \limsup _{n \rightarrow \infty} n^{-1 / \alpha} \frac{\gamma_{n-1}}{\gamma_{n}} \leqslant(2 \alpha)^{-1 / \alpha},
\end{aligned}
$$

where

$$
\lambda_{\alpha}:=\frac{\Gamma(\alpha)}{2^{\alpha-2}\{\Gamma(\alpha / 2)\}^{2}}=\frac{2^{1-\alpha} \Gamma(\alpha+1)}{\Gamma(\alpha / 2) \Gamma(\alpha / 2+1)} .
$$


Freud conjectured $[9,10]$ that for every $\alpha>0$, the sequence

$$
\left\{n^{-1 / \alpha} \frac{\gamma_{n-1}}{\gamma_{n}}\right\}_{n=1}^{\infty}
$$

actually converges and that the limit is

$$
\frac{1}{2}\left(\frac{1}{\lambda_{\alpha}}\right)^{1 / \alpha}
$$

In the well-known case of Hermite polynomials $(\alpha=2)$, the sequence (1.7) is actually a constant sequence where the constant is given by (1.8). Freud [9] proved that his conjecture is true for $\alpha=4,6$.

The validity of Freud's conjecture has several interesting consequences. Freud [10] showed how it is related to the asymptotic behavior of $X_{n}$ (the largest zero of $p_{n}$ ) and Nevai [23] utilizes the truth of Freud's conjecture when $\alpha=4$ in obtaining certain results for the polynomials $p_{n}(4 ; x)$. Furthermore, Ullman $[33,34]$ has shown how the truth of Freud's conjecture leads to the "asymptotic contracted zero distribution" for the $p_{n}$. Specifically, he proved that, assuming the truth of this conjecture, we have for every function $f$ continuous on $[-1,1]$,

$$
\lim _{n \rightarrow \infty} \frac{1}{n} \sum_{k=1}^{n} f\left(\frac{x_{k n}}{X_{n}}\right)=\int_{-1}^{1} f(x) v(x) d x,
$$

where

$$
x_{n, n}=-X_{n}<x_{n-1, n}<\cdots<x_{2, n}<X_{n}=x_{1, n}
$$

are the zeros of $p_{n}(\alpha ; x)$ (cf. [30, §3.3]) and

$$
v(x)=v(\alpha ; x):=\frac{\alpha}{\pi} \int_{|x|}^{1} \frac{y^{\alpha-1}}{\sqrt{y^{2}-x^{2}}} d y .
$$

In the present paper, we shall, on the one hand, show that the Freud conjecture is true if convergence is replaced by convergence in a certain Cesàro sense (cf. Theorems 3.4 and 3.5) and, on the other hand, obtain a version of Ullman's theorem where the validity of Freud's conjecture is not assumed (cf. Theorem 3.7).

Two additional facts emerge during our investigation which we feel are of interest in their own right.

Freud [5] proved that if $P_{n} \in \mathscr{P}_{n}, \varepsilon>0$ is given and

$$
\exp \left(-x^{2}\right)\left|P_{n}(x)\right| \leqslant M \text { for }|x| \leqslant(1+\varepsilon) \sqrt{n},
$$

then there is a constant $c(\varepsilon)$ such that

$$
\exp \left(-x^{2}\right)\left|P_{n}(x)\right| \leqslant c(\varepsilon) M \text { for }-\infty<x<\infty .
$$

Freud did not obtain sharp estimates for the constant $c(\varepsilon)$ in (1.13), but he did prove that, in a certain limiting sense, inequality (1.13) is best possible. As a special case of Theorem 2.7 of this paper, we give a substantial improvement of this result. In particular, we show that $\exp \left(-x^{2}\right)\left|P_{n}(x)\right|, x \in \mathbf{R}$, attains its maximum on the interval 
$[-\sqrt{n}, \sqrt{n}]$. And, more generally, for each $\alpha>0, \exp \left(-|x|^{\alpha}\right)\left|P_{n}(x)\right|$ attains its maximum on $\left[-a_{n}(\alpha), a_{n}(\alpha)\right]$, where

$$
a_{n}(\alpha):=\left(\frac{n}{\lambda_{\alpha}}\right)^{1 / \alpha}
$$

These results also extend a theorem of Saff and Varga [29] who showed that the maximum of $\exp (-x)\left|P_{n}(x)\right|$ on $[0, \infty)$ is actually attained on $[0,2 n]$.

The other interesting feature is a weighted analogue of Nikolskii-type inequalities. S. M. Nikolskii [24] obtained certain inequalities relating different $L^{r}$-metrics of trigonometric polynomials. In [19], Mhaskar obtained analogues of these inequalities suitable for weighted $L^{r}$-norms of algebraic polynomials. These inequalities are valid for a large class of weight functions which includes $\exp \left(-|x|^{\alpha}\right)$ only if $\alpha>1$. Further, the proofs in [19] are somewhat complicated. In the present paper, we demonstrate how a very simple proof can be given using methods employed by Nevai in [22]. We then obtain estimates on the Christoffel function for the weights $\exp \left(-|x|^{\alpha}\right), \alpha>0$, and use them to extend the Nikolskii-type inequalities to include all of these weights. These estimates are perhaps not the best possible, but, as far as we know, they are the first of their kind when $0<\alpha<1$. For $\alpha \geqslant 1$, we simplify the (unpublished) method used earlier by Freud in a course which he taught on weighted approximation.

It is interesting to note that in the previous applications of these Nikolskii-type inequalities [17-19], the results were obtained first for $L^{2}$ and then translated to other norms. However, for our present purpose, it is easier to obtain $L^{\infty}$-results first, and then deduce information about the $L^{2}$-norm.

The outline of this paper is as follows. In $\S 2$, we state our main theorems for the case of the supremum norm. In $\S 3$, we state the theorems for the case of the $L^{r}$-metrics, $0<r<\infty$, and discuss their relationship with orthogonal polynomials. In $\S \S 4$ and 5, we prove the theorems stated in $\$ \S 2$ and 3 , respectively. In $\S 6$, we state and prove the Nikolskii-type inequalities.

We would like to thank Dr. M. Lachance, University of Michigan (Dearborn), and Mr. Julian Whitekus, University of South Florida, for some of the computations included in this paper.

2. Statements of results for the sup norm. In this section we deal exclusively with the supremum norm. For any interval $I$ and function $g$ defined on $I$, we set

$$
\|g\|_{I}:=\sup \{|g(x)| ; x \in I\} .
$$

A motivating influence in our investigation is the following fundamental

Question. Let $\alpha>0$ and $P_{n} \in \mathscr{P}_{n}$, with $P_{n}$ not identically zero. Then, if $\zeta \in(-\infty, \infty)$ satisfies

$$
\exp \left(-|\zeta|^{\alpha}\right)\left|P_{n}(\zeta)\right|=\left\|\exp \left(-|x|^{\alpha}\right) P_{n}(x)\right\|_{(-\infty, \infty)},
$$

how large can $|\zeta|$ be (as a function of $\alpha$ and $n$ )?

In an implicit sense, the answer is provided by the Chebyshev polynomials associated with the weight function $\exp \left(-|x|^{\alpha}\right)$ on $(-\infty, \infty)$. To be specific, for $\alpha>0$ 
and $n=1,2, \ldots$, set

$$
E_{n}(\alpha)=E_{n, \infty}(\alpha):=\inf \left\{\left\|\exp \left(-|x|^{\alpha}\right)\left(x^{n}-q_{n-1}(x)\right)\right\|_{(-\infty, \infty)} ; q_{n-1} \in \mathscr{P}_{n-1}\right\}
$$

Let

$$
T_{n}(\alpha ; x)=T_{n, \infty}(\alpha ; x)=x^{n}+\cdots \in \mathscr{P}_{n}
$$

satisfy

$$
E_{n}(\alpha)=\left\|\exp \left(-|x|^{\alpha}\right) T_{n}(\alpha ; x)\right\|_{(-\infty, \infty)},
$$

and consider the largest extreme point

$$
\xi_{n}(\alpha):=\max \left\{\xi \in \mathbf{R} ; \exp \left(-|\xi|^{\alpha}\right)\left|T_{n}(\alpha ; \xi)\right|=E_{n}(\alpha)\right\},
$$

where $\mathbf{R}=(-\infty, \infty)$. Then, from the equioscillation property for the Chebyshev problem (2.3), we shall easily prove

THEOREM 2.1 If $P_{n} \in \mathscr{P}_{n}$ and $x \in \mathbf{R}$, then

$$
\left|P_{n}(x)\right| \leqslant\left\|\exp \left(-|t|^{\alpha}\right) P_{n}(t)\right\|_{(-\infty, \infty)} \frac{\left|T_{n}(\alpha ; x)\right|}{E_{n}(\alpha)}, \quad \forall|x| \geqslant \xi_{n}(\alpha) .
$$

Consequently, if $P_{n} \not \equiv 0$ and $\zeta$ satisfies (2.2), then

$$
|\zeta| \leqslant \xi_{n}(\alpha) \text {. }
$$

To obtain estimates for $\xi_{n}(\alpha)$ we next consider the growth of exponentially weighted polynomials in the complex plane $\mathbf{C}$. For this purpose, we set

$$
\phi(z):=z+\left(z^{2}-1\right)^{1 / 2}
$$

where $\left(z^{2}-1\right)^{1 / 2}$ has branch cut $[-1,1]$ and behaves like $z$ as $z$ tends to infinity. The function $w=\phi(z)$ maps the exterior of the segment $[-1,1]$ in the $z$-plane onto the exterior of the circle $|w|=1$ in the $w$-plane. In our results, an essential role is played by the function

$$
\begin{array}{r}
G(\alpha ; z):=\exp \left(\lambda_{\alpha}\left\{\int_{-1}^{1} \log |z-t| v(\alpha ; t) d t+\log 2+\frac{1}{\alpha}-\log |\phi(z)|-\frac{|z|^{\alpha}}{\lambda_{\alpha}}\right\}\right), \\
(\alpha>0 ; z \in \mathbf{C}),
\end{array}
$$

where $\lambda_{\alpha}$ is the constant arising in the Freud conjecture discussed in $\S 1$, i.e.

$$
\lambda_{\alpha}:=\frac{\Gamma(\alpha)}{2^{\alpha-2}\{\Gamma(\alpha / 2)\}^{2}}
$$

and $v(\alpha ; t)$ is the Ullman distribution

$$
v(\alpha ; t):=\frac{\alpha}{\pi} \int_{|t|}^{1} y^{\alpha-1} / \sqrt{y^{2}-t^{2}} d y, \quad-1 \leqslant t \leqslant 1 .
$$

Introducing the positive parameter $a$, we use the maximum principle for subharmonic functions to prove the following. 
THEOREM 2.2. Let $\alpha>0, a>0$, and suppose that $P_{n} \in \mathscr{P}_{n}$ satisfies

$$
\left\|\exp \left(-|x|^{\alpha}\right) P_{n}(x)\right\|_{[-a, a]} \leqslant 1 \text {. }
$$

Then,

$$
\exp \left(-|z|^{\alpha}\right)\left|P_{n}(z)\right| \leqslant\left\{G\left(\alpha ; \frac{z}{a}\right)\right\}^{a^{\alpha}}\left|\phi\left(\frac{z}{a}\right)\right|^{n} \quad \forall z \in \mathbf{C},
$$

where $\phi$ and $G$ are given, respectively, by (2.8) and (2.9).

We pause in our development to mention two basic properties of the function $G(\alpha ; z)$.

Proposition 2.3. For each $\alpha>0$, the function $G(\alpha ; z)$ is continuous in the whole plane, and

$$
G(\alpha ; x)=1 \text { for }-1 \leqslant x \leqslant 1 .
$$

We remark that since, also, $|\phi(x)|=1$ for $-1 \leqslant x \leqslant 1$, then the upper bound in (2.13) satisfies

$$
\{G(\alpha ; x / a)\}^{a^{\alpha}}|\phi(x / a)|^{n}=1 \text { for }-a \leqslant x \leqslant a .
$$

The function $G(\alpha ; z)$ has a useful representation in terms of the Gaussian hypergeometric function [2]

$$
F(a, b ; c ; z)=\sum_{j=0}^{\infty} \frac{(a)_{j}(b)_{j}}{(c)_{j} j !} z^{j}, \quad \begin{aligned}
& (s)_{j}:=s(s+1) \cdots(s+j-1), j \geqslant 1, \\
& (s)_{0}:=1 .
\end{aligned}
$$

Namely, we shall prove

Proposition 2.4. For each $\alpha>0$,

$$
G(\alpha ; z)=\left|\exp \left\{\mathcal{H}(\alpha ; \phi(z))-|z|^{\alpha}\right\}\right| \quad \forall z \in \mathbf{C}-[-1,1],
$$

where

$$
\mathcal{H}(\alpha ; w):=\frac{\Gamma(\alpha+1)}{2^{\alpha-1}\{\Gamma(\alpha / 2+1)\}^{2}} F\left(-\alpha / 2,1 ; \alpha / 2+1,-w^{-2}\right)-\frac{\Gamma(\alpha+1)}{2^{\alpha}\{\Gamma(\alpha / 2+1)\}^{2}} .
$$

In the case when $\alpha$ is a positive even integer, say $\alpha=2 k(k=1,2, \ldots)$, the hypergeometric function in (2.18) reduces to a polynomial in $w^{-2}$ and we find the explicit representation

$$
G(2 k ; z)=\left|\exp \left\{z^{2 k}-\left(z^{2}-1\right)^{1 / 2} g_{2 k-1}(z)-|z|^{2 k}\right\}\right| \quad(k=1,2, \ldots),
$$

where

$$
g_{1}(z):=z, \quad g_{2 k-1}(z):=z^{2 k-1}+\sum_{j=1}^{k-1}\left(\begin{array}{c}
2 j-1 \\
j
\end{array}\right) \frac{z^{2 k-2 j-1}}{2^{2 j-1}} \quad(k>1) .
$$

In Theorem 2.2, we wish to choose the parameter $a$ in an optimal fashion. It is fortuitous that two different approaches to this optimality lead to the same choice for $a$. First, on taking $P_{n}(x)=T_{n}(\alpha ; x) / E_{n}(\alpha)$ in Theorem 2.2 and letting $z \rightarrow \infty$ in 
(2.13), we obtain (cf. (2.17))

$$
E_{n}(\alpha) \geqslant \frac{a^{n}}{2^{n} \exp \left(\lambda_{\alpha} a^{\alpha} / \alpha\right)} \quad(a>0, n=1,2, \ldots) .
$$

For fixed $n$ and $\alpha$, the lower bound in (2.21) is maximized when

$$
a=\left(n / \lambda_{\alpha}\right)^{1 / \alpha} \text {. }
$$

Hence, with this choice, (2.21) yields

COROLlaRy 2.5. For each $\alpha>0$,

$$
n^{-1 / \alpha}\left[E_{n}(\alpha)\right]^{1 / n} \geqslant \frac{1}{2}\left(\frac{1}{e \lambda_{\alpha}}\right)^{1 / \alpha}, \quad n=1,2, \ldots,
$$

where $\lambda_{\alpha}$ is given in (2.10).

To numerically illustrate Corollary 2.5 we have listed in Table 1 the values $E_{n}(\alpha)$ for $\alpha=4, n=1,2, \ldots, 9$, together with the corresponding values $n^{-1 / 4}\left[E_{n}(4)\right]^{1 / n}$. From inequality (2.23) we know that for each $n \geqslant 1$,

$$
n^{-1 / 4}\left[E_{n}(4)\right]^{1 / n} \geqslant \frac{1}{2}\left(\frac{1}{e \lambda_{4}}\right)^{1 / 4}=\frac{1}{2}\left(\frac{2}{3 e}\right)^{1 / 4} \doteqdot 0.351863 ;
$$

this is clearly evident from the last column of Table 1. In fact, we shall show in Theorem 2.9 below that, for each fixed $\alpha>0$, the estimate (2.23) is best possible in the sense that equality holds in the limit as $n \rightarrow \infty$.

TABLE 1. $w(x)=\exp \left(-x^{4}\right)$

\begin{tabular}{|c|c|c|}
\hline$n$ & $E_{n}(4)$ & $n^{-1 / 4}\left[E_{n}(4)\right]^{1 / n}$ \\
\hline 1 & 0.550695 & 0.550695 \\
2 & 0.277859 & 0.443256 \\
3 & 0.163591 & 0.415566 \\
4 & 0.102626 & 0.400221 \\
5 & 0.068412 & 0.391097 \\
6 & 0.047782 & 0.384893 \\
7 & 0.034736 & 0.380422 \\
8 & 0.026136 & 0.377037 \\
9 & 0.020271 & 0.374382 \\
\hline
\end{tabular}

Returning to the discussion of the parameter $a$ in Theorem 2.2, we now describe the second optimality approach. Notice that the upper bound in (2.13) decays exponentially as $z \rightarrow \infty$. Our interest is in the smallest value for the parameter $a$ which ensures that this upper bound is less than one whenever $x \in \mathbf{R}$ and $|x|>a$. We prove

THEOREM 2.6. If $\alpha>0$ and $a \geqslant\left(n / \lambda_{\alpha}\right)^{1 / \alpha}$, then

$$
\left\{G\left(\alpha ; \frac{x}{a}\right)\right\}^{a^{\alpha}}\left|\phi\left(\frac{x}{a}\right)\right|^{n}<1 \quad \forall|x|>a, x \in \mathbf{R} .
$$

Moreover, $\left(n / \lambda_{\alpha}\right)^{1 / \alpha}$ is the smallest value of a for which (2.24) is valid. 
Next, consider the level curve

$$
\Omega(\alpha):=\left\{z \in \mathbf{C} ;[G(\alpha ; z)]^{1 / \lambda_{\alpha}}|\phi(z)|=1\right\},
$$

which is symmetric about the real axis and contains the segment $-1 \leqslant x \leqslant 1$ (cf. (2.14)). Let $\mathscr{D}(\alpha)$ denote the unbounded component of the complement $\mathbf{C}-\Omega(\alpha)$. Then, on combining Theorems 2.2 and 2.6 (with $a=\left(n / \lambda_{\alpha}\right)^{1 / \alpha}$ ), we obtain

THEOREM 2.7. If $\alpha>0$ and $P_{n}(\not \equiv 0) \in \mathscr{P}_{n}, n \geqslant 1$, satisfies

$$
\left\|\exp \left(-|x|^{\alpha}\right) P_{n}(x)\right\|_{\left[-a_{n}(\alpha), a_{n}(\alpha)\right]} \leqslant M,
$$

where

$$
a_{n}(\alpha):=\left(n / \lambda_{\alpha}\right)^{1 / \alpha}
$$

then

(2.28) $\exp \left(-|z|^{\alpha}\right)\left|P_{n}(z)\right|<M \quad \forall z \in a_{n}(\alpha) \mathscr{D}(\alpha):=\left\{z=a_{n}(\alpha) w ; w \in \mathscr{D}(\alpha)\right\}$.

In particular,

$$
\exp \left(-|x|^{\alpha}\right)\left|P_{n}(x)\right|<M \quad \forall|x|>a_{n}(\alpha), x \in \mathbf{R} .
$$

Consequently, for any $P_{n} \in \mathscr{P}_{n}$,

$$
\left\|\exp \left(-|x|^{\alpha}\right) P_{n}(x)\right\|_{\left[-a_{n}(\alpha), a_{n}(\alpha)\right]}=\left\|\exp \left(-|x|^{\alpha}\right) P_{n}(x)\right\|_{(-\infty, \infty)} .
$$

For example, since $\lambda_{1}=2 / \pi, \lambda_{2}=1$ and $\lambda_{4}=3 / 2$ (cf. (2.10)), then, for $P_{n} \in \mathscr{P}_{n}$, equation (2.30) gives as special cases:

$$
\begin{gathered}
\left\|\exp (-|x|) P_{n}(x)\right\|_{[-\pi n / 2, \pi n / 2]}=\left\|\exp (-|x|) P_{n}(x)\right\|_{(-\infty, \infty)}, \\
\left\|\exp \left(-x^{2}\right) P_{n}(x)\right\|_{[-\sqrt{n}, \sqrt{n}]}=\left\|\exp \left(-x^{2}\right) P_{n}(x)\right\|_{(-\infty, \infty)}, \\
\left\|\exp \left(-x^{4}\right) P_{n}(x)\right\|_{[-\sqrt[4]{2 n / 3}, \sqrt[4]{2 n / 3}]}=\left\|\exp \left(-x^{4}\right) P_{n}(x)\right\|_{(-\infty, \infty)} .
\end{gathered}
$$

We remark that equation (2.32) considerably improves the result of Freud mentioned in (1.12) and (1.13) of the introduction. Moreover, inequality (2.28) (with $\alpha=2$ ) provides an extension of this result to the complex plane, as illustrated in Figure 1.

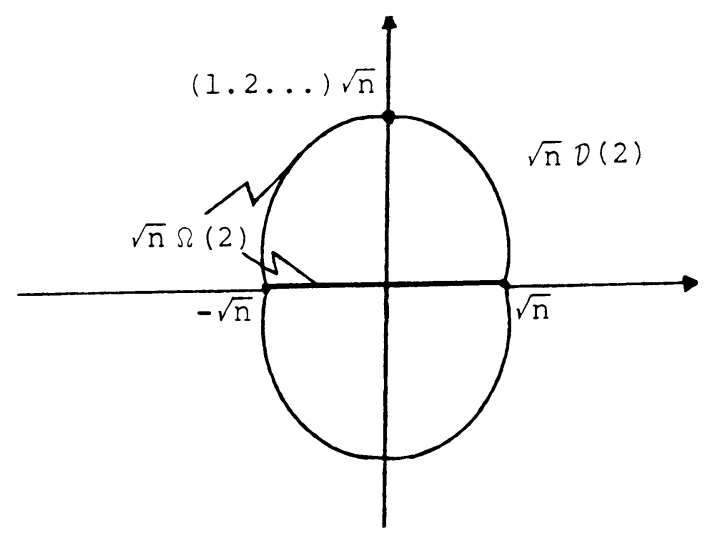

FigURE 1. $\exp \left(-|z|^{2}\right)\left|P_{n}(z)\right|<M, \forall z \in \sqrt{n} \mathcal{Q}(2)$ 
Applying Theorem 2.7 to the extremal polynomials $T_{n}(\alpha ; x)$ we obtain

COROLlaRy 2.8. The extreme point $\xi_{n}(\alpha)$ defined in (2.5) satisfies

$$
\xi_{n}(\alpha) \leqslant\left(n / \lambda_{\alpha}\right)^{1 / \alpha}=a_{n}(\alpha), \quad n=1,2, \ldots
$$

Furthermore (cf. (2.13)), for each $n=1,2, \ldots$,

$$
\begin{aligned}
& \exp \left(-\left|a_{n}(\alpha) z\right|^{\alpha}\right)\left|T_{n}\left(\alpha ; a_{n}(\alpha) z\right)\right| \leqslant E_{n}(\alpha)\left[G(\alpha ; z)^{1 / \lambda_{\alpha}}|\phi(z)|\right]^{n}, \\
& \quad \exp \left(-\left|a_{n}(\alpha) z\right|^{\alpha}\right)\left|T_{n}\left(\alpha ; a_{n}(\alpha) z\right)\right|<E_{n}(\alpha) \quad \forall z \in \mathcal{D}(\alpha) .
\end{aligned}
$$

In Theorem 2.12 we shall show that inequality (2.35) is sharp in a certain limiting sense. To numerically illustrate the inequalities (2.34) we have listed in Table 2 the values $\xi_{n}(\alpha)$ for $\alpha=4, n=1,2, \ldots, 9$. From (2.34) we know that $\xi_{n}(\alpha) / a_{n}(\alpha) \leqslant 1$ for all $n$; this is clearly confirmed in the last column of Table 2. Moreover, the numerical results suggest that $\xi_{n}(\alpha) / a_{n}(\alpha) \rightarrow 1$ as $n \rightarrow \infty$, which is a fact we shall prove in Corollary 2.11.

$$
\text { TABLE 2. } w(x)=\exp \left(-x^{4}\right)
$$

\begin{tabular}{|c|c|c|c|}
\hline$n$ & $\xi_{n}(4)$ & $a_{n}(4)=(2 n / 3)^{1 / 4}$ & $\xi_{n}(4) / a_{n}(4)$ \\
\hline 1 & 0.707107 & 0.903602 & 0.782543 \\
2 & 0.927122 & 1.074570 & 0.862784 \\
3 & 1.062582 & 1.189207 & 0.893521 \\
4 & 1.165110 & 1.277886 & 0.911748 \\
5 & 1.248090 & 1.351200 & 0.923690 \\
6 & 1.318456 & 1.414214 & 0.932289 \\
7 & 1.379846 & 1.469778 & 0.938813 \\
8 & 1.434514 & 1.519672 & 0.943963 \\
9 & 1.483938 & 1.565085 & 0.948152 \\
\hline
\end{tabular}

Using the function $G(\alpha ; x)$, we shall show that for each $\varepsilon>0$, it is possible to construct a sequence of polynomials $P_{n, \alpha}(x)=x^{n}+\cdots \in \mathscr{P}_{n}, n=1,2, \ldots$, for which

$$
\limsup _{n \rightarrow \infty} n^{-1 / \alpha}\left\|\exp \left(-|x|^{\alpha}\right) P_{n, \alpha}(x)\right\|_{\left[-a_{n}(\alpha), a_{n}(\alpha)\right]}^{1 / n} \leqslant \frac{1}{2}\left(\frac{1}{e \lambda_{\alpha}}\right)^{1 / \alpha} e^{\varepsilon}
$$

By (2.30), the norm over $\left[-a_{n}(\alpha), a_{n}(\alpha)\right]$ in (2.37) can be replaced by the norm over $(-\infty, \infty)$. Hence (2.37) together with the lower estimates of Corollary 2.5 give

THEOREM 2.9. For each $\alpha>0$,

$$
\lim _{n \rightarrow \infty} n^{-1 / \alpha}\left[E_{n}(\alpha)\right]^{1 / n}=\frac{1}{2}\left(\frac{1}{e \lambda_{\alpha}}\right)^{1 / \alpha}
$$


Theorem 2.9 is useful in establishing certain asymptotic properties of the extremal polynomials $T_{n}(\alpha ; x)$. Specifically, we note that since $\xi_{n}(\alpha) \leqslant a_{n}(\alpha)$ (cf. (2.34)) and since $T_{n}(\alpha ; x)$ has $n+1$ oscillations in the interval $|x| \leqslant \xi_{n}(\alpha)$ (cf. (4.2)), then the zeros of $T_{n}(\alpha ; x)$ all lie in the interval $-a_{n}(\alpha) \leqslant x \leqslant a_{n}(\alpha)$. Hence $T_{n}\left(\alpha ; a_{n}(\alpha) x\right)$ has all its zeros in $-1 \leqslant x \leqslant 1$. Concerning the distribution of these "contracted zeros", we prove

TheOREM 2.10. Let $\alpha>0$ be fixed. For any interval $[c, d]$ and for each $n=1,2, \ldots$, let $\Re_{n}([c, d])$ denote the number of zeros of the normalized polynomial $T_{n}\left(\alpha ;\left(n / \lambda_{\alpha}\right)^{1 / \alpha} x\right)$ which lie in $[c, d]$. Then

$$
\lim _{n \rightarrow \infty} n^{-1} \mathcal{X}_{n}([c, d])=\int_{c}^{d} v(\alpha ; t) d t
$$

where $v(\alpha ; t)$ is the Ullman distribution (2.11).

As an immediate consequence of (2.39) we see that the zeros of the sequence $\left\{T_{n}\left(\alpha ; a_{n}(\alpha) x\right)\right\}_{n=1}^{\infty}$ are dense in the interval $-1 \leqslant x \leqslant 1$. Since the (largest) extreme point $\xi_{n}(\alpha)$ is bounded above by $a_{n}(\alpha)$ and bounded below by the largest zero of $T_{n}(\alpha ; x)$, we therefore obtain

COROLlary 2.11. For each $\alpha>0$, the extreme points $\xi_{n}(\alpha)$ of (2.5) satisfy

$$
\lim _{n \rightarrow \infty}\left(\frac{n}{\lambda_{\alpha}}\right)^{-1 / \alpha} \xi_{n}(\alpha)=1 \text {. }
$$

As the final result of this section, we state the asymptotic sharpness of inequality (2.35) of Corollary 2.8.

THEOREM 2.12. The extremal polynomials $T_{n}(\alpha ; z)$ satisfy

$$
\lim _{n \rightarrow \infty} n^{-1 / \alpha}\left|\exp \left(-\left|a_{n}(\alpha) z\right|^{\alpha}\right) T_{n}\left(\alpha ; a_{n}(\alpha) z\right)\right|^{1 / n}=\frac{1}{2}\left(\frac{1}{e \lambda_{\alpha}}\right)^{1 / \alpha} G(\alpha ; z)^{1 / \lambda_{\alpha}}|\phi(z)|,
$$

uniformly for $z$ on every compact subset of $\mathbf{C}-[-1,1]$.

We remark that, from the definitions (2.9) and (2.27), the equation (2.41) can be written in the simpler form

$$
\lim _{n \rightarrow \infty}\left(\frac{n}{\lambda_{\alpha}}\right)^{-1 / \alpha}\left|T_{n}\left(\alpha ;\left(n / \lambda_{\alpha}\right)^{1 / \alpha} z\right)\right|^{1 / n}=\exp \left(\int_{-1}^{1} \log |z-t| v(\alpha ; t) d t\right) .
$$

3. Statements of results for the $L^{r}$-metrics. In this section, we discuss the extremal problems in metrics other than the sup norm. For any interval $I, r>0$ and a Lebesgue measurable function $g$ on $I$, we set

$$
\|g\|_{r, I}:= \begin{cases}\left(\int_{I}|g(x)|^{r} d x\right)^{1 / r}, & \text { if } 0<r<\infty, \\ \operatorname{ess} \sup _{I}|g(x)|, & \text { if } r=\infty\end{cases}
$$

Thus, in this notation, $\|g\|_{I}$ of (2.1) becomes $\|g\|_{\infty, I}$. Sometimes, we shall omit the suffix $\infty$ when we do not need to emphasize it. Similarly, if the interval $I$ is 
$(-\infty, \infty)$, the suffix $I$ will also be omitted. The space $L^{r}(I)$ consists of Lebesgue measurable functions $g$ on $I$ for which $\|g\|_{r, I}$ is finite; where two functions are identified if they are equal almost everywhere. Regarding the various constants which will appear in the sequel, we adopt the following convention: We shall denote the constants (depending only on $\alpha$ and the metrics involved) by $c_{1}, c_{2}$, etc. However, the same symbol may denote different constants in different formulas. Constants denoted by capital letters, however, retain their value when referred to in different formulas.

As mentioned in the introduction, Nikolskii-type inequalities play an important role in these investigations. We shall state and prove these in a very general form in $\S 6$, but for the convenience of the reader, we summarize them in the following theorem as they apply to the weight functions $\exp \left(-|x|^{\alpha}\right), \alpha>0$. Then

TheOREM 3.1. Suppose $0<p<r \leqslant \infty, \alpha>0, n \geqslant 2$ is an integer and $P_{n} \in \mathscr{P}_{n}$.

$$
\left\|w_{\alpha} P_{n}\right\|_{r} \leqslant c_{1}\left(n^{\rho}(\log n)^{\delta}\right)^{(1 / p-1 / r)}\left\|w_{\alpha} P_{n}\right\|_{p}
$$

where

$$
\begin{aligned}
& w_{\alpha}(x):=\exp \left(-|x|^{\alpha}\right), \quad x \in \mathbf{R}, \\
& \rho:= \begin{cases}1, \quad \text { if } 0<\alpha<1, \\
1-1 / \alpha, & \text { if } \alpha \geqslant 1,\end{cases} \\
& \delta:= \begin{cases}0, & \text { if } 0<\alpha<1 \text { or } \alpha \geqslant 2, \\
1, & \text { if } 1 \leqslant \alpha<2 .\end{cases}
\end{aligned}
$$

In the opposite direction,

$$
\left\|w_{\alpha} P_{n}\right\|_{p} \leqslant c_{1}\left(n^{1 / \alpha}\right)^{(1 / p-1 / r)}\left\|w_{\alpha} P_{n}\right\|_{r} .
$$

In particular, for arbitrary $p, r$ with $0<p, r \leqslant \infty$,

$$
\left\|w_{\alpha} P_{n}\right\|_{p} \leqslant c_{1} n^{c_{2}}\left\|w_{\alpha} P_{n}\right\|_{r} \leqslant c_{3} n^{c_{4}}\left\|w_{\alpha} P_{n}\right\|_{p} .
$$

In (2.30) of Theorem 2.7, we have seen that if $P_{n} \in \mathscr{P}_{n}$,

$$
\left\|w_{\alpha} P_{n}\right\|_{r,\left[-a_{n}(\alpha), a_{n}(\alpha)\right]}=\left\|w_{\alpha} P_{n}\right\|_{r},
$$

where $r=\infty$ and $a_{n}(\alpha)$ is of the order of magnitude $n^{1 / \alpha}$. Clearly, this statement cannot be true if $r<\infty$; but it is natural to expect that $\left\|w_{\alpha} P_{n}\right\|_{r}$ is at least substantially attained on an interval of length proportional to $n^{1 / \alpha}$. The following proposition makes this idea more precise.

Proposition 3.2. Suppose $\alpha>0,0<r \leqslant \infty, n \geqslant 2$ is an integer and $P_{n} \in \mathscr{P}_{n}$. Then there are positive constants $c_{1}, c_{2}$ and $D$ depending only on $r$ and $\alpha$ such that

$$
\left\|w_{\alpha} P_{n}\right\|_{r} \leqslant\left(1+c_{1} e^{-c_{2} n}\right)\left\|w_{\alpha} P_{n}\right\|_{r,\left[-D n^{1 / \alpha}, D n^{1 / \alpha}\right]} .
$$

For $\alpha \geqslant 1$ and $r=2$, inequality (3.8) was proved by Nevai [21] and Freud [8]. The proposition is easy to obtain using their ideas. In $\S 6$, we shall give an equally simple proof using the results in $\$ 2$. 
Next, we turn to Theorem 2.9. Recall from $\$ 1$ that

$$
\begin{gathered}
E_{n, r}(\alpha):=\inf _{q_{n-1} \in \mathscr{P}_{n-1}}\left\|w_{\alpha}(x)\left(x^{n}-q_{n-1}(x)\right)\right\|_{r}, \\
\left\|w_{\alpha}(x) T_{n, r}(\alpha ; x)\right\|_{r}=E_{n, r}(\alpha), \quad T_{n, r}(\alpha ; x)=x^{n}+\cdots \in \mathscr{P}_{n} .
\end{gathered}
$$

An immediate consequence of Theorem 3.1 and Theorem 2.9 is the following.

THEOREM 3.3. For each $\alpha>0$ and $r>0$,

$$
\lim _{n \rightarrow \infty} n^{-1 / \alpha}\left[E_{n, r}(\alpha)\right]^{1 / n}=\frac{1}{2}\left(\frac{1}{e \lambda_{\alpha}}\right)^{1 / \alpha} .
$$

Moreover, for each $0<p \leqslant \infty$,

$$
\lim _{n \rightarrow \infty} n^{-1 / \alpha}\left[\left\|w_{\alpha}(x) T_{n, r}(\alpha ; x)\right\|_{p}\right]^{1 / n}=\frac{1}{2}\left(\frac{1}{e \lambda_{\alpha}}\right)^{1 / \alpha} .
$$

This theorem has interesting consequences in the theory of orthogonal polynomials, which we shall now discuss. As we mentioned in the introduction, if

$$
p_{n}(\alpha ; x):=\gamma_{n}(\alpha) x^{n}+\cdots \in \mathscr{P}_{n}, \quad \gamma_{n}(\alpha)>0,
$$

are orthonormal polynomials with respect to $w_{\alpha}^{2}$, then

$$
E_{n, 2}(\alpha)=\frac{1}{\gamma_{n}(\alpha)}, \quad T_{n, 2}(\alpha ; x)=\frac{1}{\gamma_{n}(\alpha)} p_{n}(\alpha ; x) .
$$

Theorem 3.3 applied to $r=2$ thus gives information about the asymptotic behavior of $\gamma_{n}(\alpha)$. In order to study the implications of this theorem related to the Freud conjecture, we shall reformulate the latter as follows.

Set

$$
\begin{gathered}
C_{n}=C_{n}(\alpha):=\frac{\gamma_{n-1}(\alpha)}{\gamma_{n}(\alpha)}, \quad A_{n}=A_{n}(\alpha):=n^{-1 / \alpha} C_{n}(\alpha), \\
B_{n}=B_{n}(\alpha):=\log A_{n}(\alpha), \\
d_{\alpha}:=\frac{1}{2}\left(\frac{1}{\lambda_{\alpha}}\right)^{1 / \alpha} .
\end{gathered}
$$

Then the Freud conjecture states that

$$
\lim _{n \rightarrow \infty} B_{n}=\log \left\{\frac{1}{2}\left(\frac{1}{\lambda_{\alpha}}\right)^{1 / \alpha}\right\}=\log d_{\alpha} .
$$

As a consequence of Theorem 3.3 (with $r=2$ ), (3.14) and Stirling's formula, we obtain

THeOREM 3.4. For each $\alpha>0$,

$$
\lim _{n \rightarrow \infty} \frac{1}{n} \sum_{k=1}^{n} B_{k}=\log d_{\alpha},
$$

where $B_{k}$ and $d_{\alpha}$ are defined, respectively, in (3.16) and (3.17). 
REMARK. Theorem 3.4 implies that if the sequence $\left\{B_{n}\right\}_{n=1}^{\infty}$ converges, then the limit must be $\log d_{\alpha}$. When $\alpha$ is a positive even integer, this is apparent from (1.4) and (1.5); for other values of $\alpha$, this assertion is new.

Theorem 3.4 also suggests that it might be possible to use tauberian theorems to settle the Freud conjecture. While we are not yet able to do this, we can prove the following result which comes close!

THEOREM 3.5. (a) If $\alpha \geqslant 1$, the sequence $\left\{B_{n}\right\}$ converges in the Cesàro sense $(C, \varepsilon)$ for every $\varepsilon>0$.

(b) If $\alpha>0$ and if, for all $n$ large,

$$
C_{n+1} \geqslant C_{n},
$$

where $C_{n}$ is defined in (3.15), then the sequence $\left\{B_{n}\right\}$ converges to $\log d_{\alpha}$.

Numerical evidence suggests that the sequence $\left\{C_{n}\right\}$ is eventually increasing. If, indeed, this is true, then part (b) of Theorem 3.5 would imply the truth of Freud's conjecture.

The contracted zero distribution for the orthonormal polynomials $\left\{p_{n}(\alpha ; x)\right\}$ is obtained from the following general result which is analogous to Fekete's theorem (cf. [3]) for finite intervals.

THEOREM 3.6. Let $K \geqslant 1$ be fixed and consider a triangular array of (not necessarily distinct ) points in $[-K, K]$ :

$$
\left[\begin{array}{cccc}
z_{1,1} & & & \\
z_{1,2} & z_{2,2} & & \\
\vdots & \vdots & & \\
z_{1, n} & z_{2, n} & \ldots & z_{n, n} \\
\cdots & \cdots & . & \ldots
\end{array}\right] .
$$

With this array, associate the sequence of polynomials

$$
Q_{n}(x):=\prod_{k=1}^{n}\left(x-z_{k, n}\right), \quad n=1,2, \ldots,
$$

and the sequence of measures $\left\{\nu_{n}\right\}_{n=1}^{\infty}$, where for each Borel set $\mathscr{B} \subset[-K, K]$,

$$
\nu_{n}(\mathscr{B}):=\frac{\left|\left\{k: z_{k, n} \in \mathscr{B}\right\}\right|}{n}, \quad n=1,2, \ldots
$$

If

$$
\underset{n \rightarrow \infty}{\limsup }\left\{\left\|\exp \left(\frac{-n|x|^{\alpha}}{\lambda_{\alpha}}\right) Q_{n}(x)\right\|_{\infty,[-1,1]}\right\}^{1 / n} \leqslant \frac{1}{2} \exp \left(-\frac{1}{\alpha}\right),
$$

then, for every interval $[c, d] \subset[-K, K]$,

$$
\lim _{n \rightarrow \infty} \int_{c}^{d} d \nu_{n}(t)=\int_{c}^{d} v(\alpha ; t) d t
$$

where $v(\alpha ; t)$ is the Ullman distribution defined in (2.11). 
Notice that although the array (3.21) may have points outside $[-1,1]$, the inequality (3.24) involves the sup norm only on $[-1,1]$. If (3.24) holds, then as a consequence of (3.25), the proportion of knots in the $n$th row of (3.21) which lie outside $[-1,1]$ tends to zero as $n \rightarrow \infty$. This feature of Theorem 3.6 is important in describing the contracted zero distribution of the orthonormal polynomials $\left\{p_{n}(\alpha ; x)\right\}$. If $X_{n}=X_{n}(\alpha)$ denotes the largest positive zero of $p_{n}(\alpha ; x), \alpha>0$, it is known [21] that

$$
c_{1} n^{1 / \alpha} \leqslant X_{n} \leqslant c_{2} n^{1 / \alpha}, \quad n=1,2, \ldots
$$

Hence the zeros of the normalized polynomials $p_{n}\left(\alpha ;\left(n / \lambda_{\alpha}\right)^{1 / \alpha} x\right), n=1,2, \ldots$, all lie in a fixed interval

$$
-K_{\alpha} \leqslant x \leqslant K_{\alpha}, \quad K_{\alpha} \geqslant 1 .
$$

From (3.12) (with $p=\infty, r=2$ ), (3.14) and Theorem 3.6, we therefore obtain

THEOREM 3.7. For the polynomials $p_{n}(\alpha ; x)=\gamma_{n} \prod_{k=1}^{n}\left(x-x_{k, n}\right), n=1,2, \ldots$, which are orthonormal with respect to $\exp \left(-2|x|^{\alpha}\right)$ on $\mathbf{R}$, set

$$
y_{k, n}:=\left(n / \lambda_{\alpha}\right)^{-1 / \alpha} x_{k, n}, \quad k=1,2, \ldots, n ; n=1,2, \ldots
$$

For any interval $[c, d]$ and for each $n=1,2, \ldots$, let $\Re_{n, 2}([c, d])$ denote the number of the $y_{k, n}$ which lie in the interval $[c, d]$. Then

$$
\lim _{n \rightarrow \infty} n^{-1} \Re_{n, 2}([c, d])=\int_{c}^{d} v(\alpha ; t) d t,
$$

where $v(\alpha ; t)$ is the Ullman distribution (2.11).

To deduce the zero distribution for the extremal polynomials $T_{n, r}(\alpha ; x)$, where $r \neq 2, \infty$, we utilize

Proposition 3.8. Suppose $r>0$ and $\alpha>0$.

(a) For each $n=1,2, \ldots$, the polynomial $T_{n, r}(\alpha ; x)$ has $n$ real zeros. ${ }^{3}$

(b) For each $\delta>0$, let $m_{n}(\delta)$ denote the number of zeros of the normalized polynomials $T_{n, r}\left(\alpha ;\left(n / \lambda_{\alpha}\right)^{1 / \alpha} x\right)$ which lie outside the interval $[-1-\delta, 1+\delta]$. Then

$$
\lim _{n \rightarrow \infty} \frac{m_{n}(\delta)}{n}=0 .
$$

Although we have not shown that the contracted zeros of $T_{n, r}(\alpha ; x)$ satisfy the conditions of Theorem 3.6, we can, by Proposition 3.8, ignore the proportion of these zeros which lie outside of, say, $[-2,2]$. Then, with a little manipulation, we can prove that (3.25) persists and gives

THEOREM 3.9. Let $r>0$ and $\alpha>0$ be fixed. For any interval $[c, d]$ and for each $n=1,2, \ldots$, let $\Re_{n, r}([c, d])$ denote the number of zeros of the normalized polynomials $T_{n, r}\left(\alpha ;\left(n / \lambda_{\alpha}\right)^{1 / \alpha} x\right)$ which lie in $[c, d]$. Then

$$
\lim _{n \rightarrow \infty} n^{-1} \Re_{n, r}([c, d])=\int_{c}^{d} v(\alpha ; t) d t
$$

\footnotetext{
${ }^{3}$ We make no assumptions here and throughout the paper concerning the uniqueness of the $T_{n, r}(\alpha ; x)$. If $r<1$, such uniqueness is not clear; however, our results hold for any choice of $T_{n, r}(\alpha ; x)$ satisfying (3.10).
} 
Corresponding to Theorem 2.12, we have the following asymptotic formula for the extremal polynomials $T_{n, r}(\alpha ; z)$.

THEOREM 3.10. Let $r>0, \alpha>0$. Then the polynomials $T_{n, r}(\alpha ; z)$ satisfy

$$
\lim _{n \rightarrow \infty}\left(n / \lambda_{\alpha}\right)^{-1 / \alpha}\left|T_{n, r}\left(\alpha ;\left(n / \lambda_{\alpha}\right)^{1 / \alpha} z\right)\right|^{1 / n}=\exp \left(\int_{-1}^{1} \log z-t \mid v(\alpha ; t) d t\right)
$$

uniformly for $z$ on every compact subset of $\mathbf{C}-\mathbf{R}$. Moreover, if $r=2$, the convergence in (3.32) is uniform on compact subsets of $\mathbf{C}-\left[-K_{\alpha}, K_{\alpha}\right]$, where $K_{\alpha}$ is as in (3.27).

\section{Proofs of the results stated in $\$ 2$.}

Proof of Theorem 2.1. It is easily seen that the functions $\left\{\exp \left(-|x|^{\alpha}\right) x^{k}\right\}_{k=0}^{n-1}$ form a Haar system on $(-\infty, \infty)$. Hence (cf. [31]) there exist $n+1$ points

$$
\xi_{n}^{(0)}<\xi_{n}^{(1)}<\cdots<\xi_{n}^{(n)}=\xi_{n}(\alpha) \quad\left(\xi_{n}^{(k)}=\xi_{n}^{(k)}(\alpha)\right)
$$

such that

$$
\exp \left(-\left|\xi_{n}^{(k)}\right|^{\alpha}\right) T_{n}\left(\alpha ; \xi_{n}^{(k)}\right)=(-1)^{n-k} E_{n}(\alpha), \quad k=0,1, \ldots, n .
$$

Because of the symmetry of the weight function, we can take $\xi_{n}^{(0)}=-\xi_{n}(\alpha)$.

Since inequality (2.6) is trivially true when $P_{n} \equiv 0$, assume that $P_{n} \in \mathscr{P}_{n}$ and $P_{n} \not \equiv 0$. Then, for any constant $\gamma$ satisfying

$$
|\gamma|<E_{n}(\alpha) /\left\|\exp \left(-|x|^{\alpha}\right) P_{n}(x)\right\|_{(-\infty, \infty)}
$$

the polynomial

$$
q(x):=T_{n}(\alpha ; x)-\gamma P_{n}(x) \in \mathscr{P}_{n}
$$

has alternating signs in the points $\xi_{n}^{(k)}, k=0,1, \ldots, n$. Hence $q(x)$ has no zeros for $|x| \geqslant \xi_{n}(\alpha)$. As $q\left(\xi_{n}(\alpha)\right)>0$, then necessarily

$$
T_{n}(\alpha ; x)-\gamma P_{n}(x)>0 \text { for } x \geqslant \xi_{n}(\alpha) .
$$

On letting $|\gamma|$ tend to the right-hand side of (4.3), inequality (4.4) yields (2.6) for $x \geqslant \xi_{n}(\alpha)$. The case when $x \leqslant-\xi_{n}(\alpha)$ is treated similarly.

The proof of Theorem 2.2 requires a few preliminary results. The first lemma appears in [14, p. 69].

LEMMA 4.1. Let $\mu$ be a finite (positive) measure on the Borel subsets of $[-1,1]$ and set

$$
\Lambda(d \mu ; z):=\int_{-1}^{1} \log |z-t| d \mu(t), \quad z \in \mathbf{C} .
$$

If $\Lambda(d \mu ; x)$ is a continuous function of a real variable on $[-1,1]$, then $\Lambda(d \mu ; z)$ is a continuous function of a complex variable on the whole plane $\mathbf{C}$.

In computing the logarithmic potential of the Ullman distribution (2.11) we make use of the following identity.

LEMMA 4.2. For all $z \in \mathbf{C}$,

$$
\frac{1}{\pi} \int_{-1}^{1} \log |z-t| d t / \sqrt{1-t^{2}}=\log \left|\frac{\phi(z)}{2}\right|,
$$

where $\phi(z)$ is defined in (2.8). 
ProOF. Write

$$
z=\frac{1}{2}\left(w+w^{-1}\right), \quad|w| \leqslant 1 .
$$

Then, with the change of variable $t=\cos \theta$, we obtain

$$
\begin{aligned}
\frac{1}{\pi} \int_{-1}^{1} \log |z-t| d t / \sqrt{1-t^{2}} & =\frac{1}{\pi} \int_{0}^{\pi} \log |z-\cos \theta| d \theta \\
& =\frac{1}{\pi} \int_{0}^{\pi} \log \left|\frac{\left(w-e^{i \theta}\right)\left(w-e^{-i \theta}\right)}{2 w}\right| d \theta \\
& =\log \left|\frac{1}{2 w}\right|+\frac{1}{\pi} \int_{-\pi}^{\pi} \log \left|1-w e^{i \theta}\right| d \theta
\end{aligned}
$$

If $|w|<1$, then by the mean value property for harmonic functions [1], the last integral in (4.7) is zero. The same is true if $|w|=1$ as is shown in [16, §35, equation (8.10)]. Hence, for all $z \in \mathbf{C}$,

$$
\frac{1}{\pi} \int_{-1}^{1} \log |z-t| \frac{d t}{\sqrt{1-t^{2}}}=\log \left|\frac{1}{2 w}\right|=\log \left|\frac{\phi(z)}{2}\right| .
$$

For the logarithmic potential of the unit measure $v(\alpha ; t) d t$ on $[-1,1]$, we can now prove

LEMMA 4.3. For $\alpha>0$ and $z \in \mathrm{C}$, set

$$
\mathcal{L}(\alpha ; z):=\int_{-1}^{1} \log z-t \mid v(\alpha ; t) d t
$$

where $v(\alpha ; t)$ is defined by (2.11). Then $\mathcal{L}(\alpha ; z)$ is a continuous function of $z$ in the whole plane C. Furthermore, for $x \in[-1,1]$,

$$
\mathcal{L}(\alpha ; x)=\frac{|x|^{\alpha}}{\lambda_{\alpha}}-\log 2-\frac{1}{\alpha},
$$

where $\lambda_{\alpha}$ is defined in (2.10).

We remark that for $\alpha$ a positive even integer, formula (4.9) was proved by Ullman [33].

Proof OF LemMa 4.3. Once we prove (4.9), the continuity assertion follows from Lemma 4.1.

To establish (4.9), first observe that, by (2.11),

$$
\mathcal{L}(\alpha ; x)=\frac{\alpha}{\pi} \int_{-1}^{1} \int_{|t|}^{1} \frac{y^{\alpha-1} \log |x-t|}{\sqrt{y^{2}-t^{2}}} d y d t .
$$

For $x \in[-1,1]$, we apply Tonelli's theorem (cf. [27]) to the integral

$$
\log 2-\mathcal{L}(\alpha ; x)=\frac{\alpha}{\pi} \int_{-1}^{1} \int_{|t|}^{1} \frac{y^{\alpha-1}(\log 2-\log |x-t|)}{\sqrt{y^{2}-t^{2}}} d y d t,
$$

and deduce that

$$
\mathcal{L}(\alpha ; x)=\frac{\alpha}{\pi} \int_{0}^{1} \int_{-y}^{y} \frac{y^{\alpha-1} \log |x-t|}{\sqrt{y^{2}-t^{2}}} d t d y
$$


In view of the identity (4.6), we therefore have

$$
\begin{aligned}
\mathcal{L}(\alpha ; x) & =\alpha \int_{0}^{1} y^{\alpha-1} \log \left|\frac{y \phi(x / y)}{2}\right| d y \\
& =-\log 2+I_{1}+I_{2},
\end{aligned}
$$

where

$$
\begin{gathered}
I_{1}:=\alpha \int_{0}^{|x|} y^{\alpha-1} \log |y \phi(x / y)| d y, \\
I_{2}:=\alpha \int_{|x|}^{1} y^{\alpha-1} \log |y \phi(x / y)| d y .
\end{gathered}
$$

We first compute $I_{2}$. Notice that, from definition (2.8), we have, for $|x| \leqslant y \leqslant 1$,

$$
\log |y \phi(x / y)|=\log |y|=\log y .
$$

Hence,

$$
I_{2}=\alpha \int_{|x|}^{1} y^{\alpha-1} \log y d y=|x|^{\alpha} / \alpha-1 / \alpha-|x|^{\alpha} \log |x| .
$$

Next, a simple change of variable in (4.13) yields

$$
\begin{aligned}
I_{1} & =\alpha|x|^{\alpha} \int_{0}^{1} u^{\alpha-1} \log |x u \phi(1 / u)| d u \\
& =|x|^{\alpha} \log |x|+|x|^{\alpha} J,
\end{aligned}
$$

where

$$
J:=\alpha \int_{0}^{1} u^{\alpha-1} \log |u \phi(1 / u)| d u=\alpha \int_{0}^{1} u^{\alpha-1} \log \left(1+\sqrt{1-u^{2}}\right) d u
$$

Integrating the last integral by parts gives

$$
J=\int_{0}^{1} \frac{u^{\alpha}}{1+\sqrt{1-u^{2}}} \cdot \frac{u}{\sqrt{1-u^{2}}} d u
$$

and putting $s=\sqrt{1-u^{2}}$ yields

$$
\begin{aligned}
J & =\int_{0}^{1} \frac{\left(1-s^{2}\right)^{\alpha / 2}}{1+s} d s=\int_{0}^{1}\left(1-s^{2}\right)^{(\alpha / 2)-1}(1-s) d s \\
& =\int_{0}^{1}\left(1-s^{2}\right)^{(\alpha / 2)-1} d s-\int_{0}^{1} s\left(1-s^{2}\right)^{(\alpha / 2)-1} d s \\
& =\frac{2^{\alpha-2}\{\Gamma(\alpha / 2)\}^{2}}{\Gamma(\alpha)}-\frac{1}{\alpha}=\frac{1}{\lambda_{\alpha}}-\frac{1}{\alpha} .
\end{aligned}
$$

Thus, from (4.16), we obtain

$$
I_{1}=|x|^{\alpha} \log |x|+|x|^{\alpha} / \lambda_{\alpha}-|x|^{\alpha} / \alpha .
$$

Equation (4.9) now follows from (4.12), (4.15) and (4.19).

Proof of Theorem 2.2. The hypothesis (2.12) can be expressed as

$$
f(x):=\log \left|P_{n}(a x)\right|-a^{\alpha}|x|^{\alpha} \leqslant 0 \quad \forall x \in[-1,1] .
$$


We shall show that for all $z \in \mathbf{C}$,

$$
F(z):=\log \left|P_{n}(a z)\right|-n \log |\phi(z)|-a^{\alpha} \tilde{G}(z) \leqslant 0,
$$

where $\phi(z)$ is defined in (2.8) and

$$
\tilde{G}(z):=\lambda_{\alpha}\left\{\int_{-1}^{1} \log |z-t| v(\alpha ; t) d t+\log 2+1 / \alpha-\log |\phi(z)|\right\} .
$$

For this purpose, notice that $\tilde{G}(z)$ is harmonic in $\mathbf{C}-[-1,1]$. Also, since $v(\alpha ; t) d t$ is a unit measure and $\phi(z)$ behaves like $2 z$ as $z \rightarrow \infty$, the function $\tilde{G}(z)$ is harmonic at $z=\infty$. Furthermore, Lemma 4.3 implies that

$$
\tilde{G}(z) \rightarrow|x|^{\alpha} \quad \text { as } z \rightarrow x \in[-1,1], \quad z \in \mathbf{C}-[-1,1],
$$

and so

$$
F(z) \rightarrow f(x) \text { as } z \rightarrow x \in[-1,1], \quad z \in \mathbf{C}-[-1,1] .
$$

Finally, observe that $F(z)$ is subharmonic in $\mathrm{C}-[-1,1]$, and because $P_{n}$ is a polynomial of degree at most $n, F(z)$ is also subharmonic at infinity. Consequently, by the maximum modulus principle for subharmonic functions [16], the inequality (4.20) implies (4.21) which is equivalent to (2.13).

Proof of Proposition 2.3. With definition (2.9), the proposition is an immediate consequence of Lemma 4.3.

Proof of Proposition 2.4. Consider the analytic function $h_{\alpha}(w), \alpha>0$, defined by the Poisson integral

$$
h_{\alpha}(w):=\frac{1}{2 \pi} \int_{-\pi}^{\pi}|\cos \theta|^{\alpha}\left(\frac{1+e^{i \theta} w}{1-e^{i \theta} w}\right) d \theta=\frac{b_{0}}{2}+\sum_{k=1}^{\infty} b_{k} w^{k} .
$$

Then $h_{\alpha}(w)$ is analytic in $|w|<1$ and the real part of $h_{\alpha}(w)$ is continuous in the closed disk $|w| \leqslant 1$ and satisfies

$$
\operatorname{Re}\left\{h_{\alpha}(w)\right\}=|\cos \theta|^{\alpha}, \quad w=e^{i \theta}, 0 \leqslant \theta \leqslant 2 \pi .
$$

The coefficients $b_{k}$ in (4.25) are given by

$$
b_{k}=\frac{1}{\pi} \int_{-\pi}^{\pi}|\cos \theta|^{\alpha} e^{i k \theta} d \theta=\frac{1}{\pi} \int_{-\pi}^{\pi}|\cos \theta|^{\alpha} \cos (k \theta) d \theta, \quad k=0,1, \ldots
$$

It is easy to see, from (4.27), that

$$
b_{2 j+1}=0, \quad j=0,1, \ldots,
$$

and, by the known integral formula [2, equation (30), p. 12],

$$
b_{2 j}=\frac{\Gamma(\alpha+1)}{2^{\alpha-1} \Gamma(\alpha / 2+j+1) \Gamma(\alpha / 2-j+1)}, \quad j=0,1, \ldots
$$

Using the identity [2, equation (6), p. 3] for complementary arguments of the Gamma function, we have

$$
\frac{1}{\Gamma(\alpha / 2-j+1)}=\frac{(-1)^{j} \Gamma(j-\alpha / 2)}{\Gamma(-\alpha / 2) \Gamma(\alpha / 2+1)},
$$


and hence formula (4.29) can be written as

$$
\begin{aligned}
b_{2 j} & =\frac{\Gamma(\alpha+1)(-1)^{j} \Gamma(j-\alpha / 2)}{2^{\alpha-1} \Gamma(-\alpha / 2) \Gamma(\alpha / 2+1) \Gamma(\alpha / 2+j+1)} \\
& =(-1)^{j} \frac{\Gamma(\alpha+1)(-\alpha / 2)_{j}}{2^{\alpha-1}\{\Gamma(\alpha / 2+1)\}^{2}(\alpha / 2+1)_{j}} .
\end{aligned}
$$

Thus, from (4.25), (4.28) and (4.30), we have

$$
\begin{aligned}
h_{\alpha}(w)= & \frac{\Gamma(\alpha+1)}{2^{\alpha-1}\{\Gamma(\alpha / 2+1)\}^{2}} F\left(-\frac{\alpha}{2}, 1 ; \frac{\alpha}{2}+1 ;-w^{2}\right) \\
& -\frac{\Gamma(\alpha+1)}{2^{\alpha}\{\Gamma(\alpha / 2+1)\}^{2}} .
\end{aligned}
$$

Next, note that the function $\operatorname{Re}\left\{h_{\alpha}(1 / \phi(z))\right\}$ is continuous in $\mathbf{C}^{*}:=\mathbf{C} \cup\{\infty\}$, harmonic in $\mathbf{C}^{*}-[-1,1]$ and, by $(4.26)$, satisfies

$$
\operatorname{Re}\left\{h_{\alpha}(1 / \phi(x))\right\}=|x|^{\alpha} \quad \forall x \in[-1,1] .
$$

Since, by Lemma 4.3, the same is true for the function $\tilde{G}(z)$ of (4.22), then

$$
\operatorname{Re}\left\{h_{\alpha}(1 / \phi(z))\right\}=\tilde{G}(z) \quad \forall z \in \mathbf{C}^{*} .
$$

Equation (2.17) now follows from (4.31) and (4.32).

Proof of Theorem 2.6. For $\alpha>0$ and $a>0$, put

$$
\begin{aligned}
g_{a}(x):= & a^{\alpha} \log G(\alpha ; x)+n \log |\phi(x)| \\
= & a^{\alpha} \lambda_{\alpha}\left\{\mathcal{L}(\alpha ; x)+\log 2+1 / \alpha-|x|^{\alpha} / \lambda_{\alpha}\right\} \\
& +\left(n-a^{\alpha} \lambda_{\alpha}\right) \log |\phi(x)|, \quad x \in \mathbf{R},
\end{aligned}
$$

where $\mathcal{L}(\alpha ; x)$ is defined in (4.8). Notice that, by Proposition 2.3 , the function $g_{a}(x)$ is continuous on $\mathbf{R}$ and satisfies

$$
g_{a}(x)=0, \quad \forall x \in[-1,1]
$$

Our goal is to find the smallest value of the parameter $a$ for which $g_{a}(x)<0$ for all $|x|>1, x \in \mathbf{R}$. For this purpose we first compute the derivative of $\mathcal{L}(\alpha ; x)$.

Let $x>1$ and observe that, from the proof of Lemma 4.3 (cf. (4.12), (4.14), and (4.19)), we have

$$
\begin{aligned}
\mathcal{L}(\alpha ; x)= & -\log 2+I_{1}+I_{2} \\
= & -\log 2+x^{\alpha} \log x+x^{\alpha} / \lambda_{\alpha}-x^{\alpha} / \alpha \\
& -\alpha \int_{1}^{x} y^{\alpha-1} \log (y \phi(x / y)) d y .
\end{aligned}
$$

Since $\phi^{\prime}(u) / \phi(u)=1 / \sqrt{u^{2}-1}$ for $u>1$, it follows that

$$
\begin{aligned}
\frac{d}{d x} \mathcal{L}(\alpha ; x) & =\alpha x^{\alpha-1} \log x+\frac{\alpha x^{\alpha-1}}{\lambda_{\alpha}}-\alpha\left(x^{\alpha-1} \log x+\int_{1}^{x} \frac{y^{\alpha-1}}{\sqrt{x^{2}-y^{2}}} d y\right) \\
& =\frac{\alpha x^{\alpha-1}}{\lambda_{\alpha}}-\alpha \int_{1}^{x} \frac{y^{\alpha-1}}{\sqrt{x^{2}-y^{2}}} d y, \quad x>1 .
\end{aligned}
$$


From (4.33) and (4.36), we get

$$
\frac{d}{d x} g_{a}(x)=\frac{\left(n-a^{\alpha} \lambda_{\alpha}\right)}{\sqrt{x^{2}-1}}-\alpha a^{\alpha} \lambda_{\alpha} \int_{1}^{x} \frac{y^{\alpha-1}}{\sqrt{x^{2}-y^{2}}} d y, \quad x>1 \text {. }
$$

Consequently, if $a \geqslant\left(n / \lambda_{\alpha}\right)^{1 / \alpha}$, then $g_{a}^{\prime}(x)<0$ for $x>1$. With (4.34), this implies that, for $a \geqslant\left(n / \lambda_{\alpha}\right)^{1 / \alpha}$,

$$
g_{a}(x)<0 \quad \forall x>1,
$$

and, moreover, $\left(n / \lambda_{\alpha}\right)^{1 / \alpha}$ is the smallest value of $a$ for which (4.38) holds for all $x>1$. A similar computation shows that the last assertion is true with " $x>1$ " replaced by " $x<-1$ ". This completes the proof of Theorem 2.6.

As stated in $\$ 2$, Theorem 2.7 and Corollary 2.8 are immediate consequences of the preceding results in that section. To prove Theorem 2.9 we first establish

LEMMA 4.4. Let $\mu$ be a unit (positive) measure on the Borel sets of $[-K, K]$ and put

$$
\Lambda(d \mu ; x):=\int_{-K}^{K} \log |x-t| d \mu(t), \quad x \in \mathbf{R} .
$$

If $\Lambda(d \mu ; x)$ is a continuous function on $\mathbf{R}$, then for each $\varepsilon>0$, there exists an integer $N=N(\varepsilon)$ and a sequence of monic polynomials $P_{n}(x)=x^{n}+\cdots \in \mathscr{P}_{n}, n=N$, $N+1, \ldots$, such that

$$
\frac{1}{n} \log \left|P_{n}(x)\right| \leqslant \varepsilon+\Lambda(d \mu ; x) \quad \forall x \in[-K, K], n \geqslant N .
$$

The essential feature of Lemma 4.4 is the uniformity in $x$ of (4.40). If only pointwise estimates were required, the result would be a simple consequence of the constructive methods used by Pólya and Szegö [26] and the lower envelope theorem of potential theory (cf. [32, Lemma 4.3]).

Proof of Lemma 4.4. First we assume that $d \mu$ is of the form $d \mu(t)=f(t) d t$, where $f(t)$ is a nonnegative continuous function on $[-K, K]$ with

$$
\int_{-K}^{K} f(t) d t=1
$$

For each $R>0$, define

$$
\log _{R}|x|:=\max (\log |x|,-R) .
$$

Note that, for $x \in[-K, K]$,

$$
\left|\int_{-K}^{K} \log \right| x-t\left|f(t) d t-\int_{-K}^{K} \log _{R}\right| x-t|f(t) d t| \leqslant 2 M \int_{0}^{e^{-R}} \log u \mid d u<\infty,
$$

where $M:=\max \{f(x): x \in[-K, K]\}$. Given $\varepsilon>0$, we can therefore find an $R=$ $R(\varepsilon)>0$ such that for every $x \in[-K, K]$,

$$
\int_{-K}^{K} \log _{R}|x-t| f(t) d t \leqslant \frac{\varepsilon}{3}+\Lambda(d \mu ; x) .
$$

Next, for each $n \geqslant 1$, select points $\left\{y_{j, n}\right\}_{j=0}^{n}$ such that

$$
-K=y_{0, n}<y_{1, n}<\cdots<y_{n, n}=K,
$$


and

$$
\int_{y_{j, n}}^{y_{j+1, n}} f(t) d t=\frac{1}{n}, \quad j=0,1, \ldots, n-1 .
$$

In view of (4.43) and the fact that

$\left|\log _{R}\right| x-t\left|-\log _{R}\right| x-y_{j, n}|| \leqslant e^{R}\left|y_{j+1, n}-y_{j, n}\right| \quad \forall t \in\left[y_{j, n}, y_{j+1, n}\right], x \in[-K, K]$, it is easy to verify that

$$
\left|\int_{-K}^{K} \log _{R}\right| x-t\left|f(t) d t-\frac{1}{n} \sum_{j=0}^{n-1} \log _{R}\right| x-y_{j, n}|| \leqslant \frac{2 K e^{R}}{n} \quad \forall x \in[-K, K] .
$$

Select $N=N(\varepsilon)$ such that for $n \geqslant N, 2 K e^{R} / n<\varepsilon / 3$, where $R=R(\varepsilon)$. Then, by (4.42) and (4.44), we have

$$
\begin{aligned}
\frac{2 \varepsilon}{3}+\Lambda(d \mu ; x) & \geqslant \frac{1}{n} \sum_{j=0}^{n-1} \log _{R}\left|x-y_{j, n}\right| \\
& \geqslant \frac{1}{n} \sum_{j=0}^{n-1} \log \left|x-y_{j, n}\right| \quad \forall x \in[-K, K], n \geqslant N .
\end{aligned}
$$

This proves Lemma 4.4 when $d \mu(t)=f(t) d t$ with $f(t)$ continuous on $[-K, K]$.

For the general case, set

$$
\psi(t):= \begin{cases}\exp \left(t^{2} /\left(t^{2}-1\right)\right) / \sigma, & \text { for } 0 \leqslant|t|<1 \\ 0, & \text { for } 1 \leqslant|t|\end{cases}
$$

where the constant $\sigma$ is chosen so that

$$
\int_{-1}^{1} \psi(t) d t=1
$$

Next, for $\delta>0$, let

$$
f_{\delta}(x):=\frac{1}{\delta} \int_{-K}^{K} \psi\left(\frac{x-s}{\delta}\right) d \mu(s) .
$$

Then $f_{\delta}$ is clearly a nonnegative continuous function with support in $[-K-\delta, K+\delta]$ and

$$
\int_{-K-\delta}^{K+\delta} f_{\delta}(x) d x=1 .
$$

For a given $\varepsilon>0$, the uniform continuity of $\Lambda(d \mu ; x)$ on the interval [ $-K-1$, $K+1]$ implies that there exists a $\delta_{\varepsilon}$, with $0<\delta_{\varepsilon}<1$, such that for all $x \in[-K, K]$ and for all $y \in\left[-\delta_{\varepsilon}, \delta_{\varepsilon}\right]$,

$$
\left|\int_{-K}^{K} \log \right| x-s-y\left|d \mu(s)-\int_{-K}^{K} \log \right| x-s|d \mu(s)|<\frac{\varepsilon}{3} .
$$


Furthermore, by using Tonelli's theorem, we can write

$$
\begin{aligned}
\int_{-K-\delta}^{K+\delta} \log |x-t| f_{\delta}(t) d t & =\frac{1}{\delta} \int_{-K-\delta}^{K+\delta} \int_{-K}^{K} \log |x-t| \psi\left(\frac{t-s}{\delta}\right) d \mu(s) d t \\
& =\frac{1}{\delta} \int_{-K}^{K} \int_{|t-s| \leqslant \delta} \log |x-t| \psi\left(\frac{t-s}{\delta}\right) d t d \mu(s) \\
& =\frac{1}{\delta} \int_{-K}^{K} \int_{-\delta}^{\delta} \log |x-s-y| \psi\left(\frac{y}{\delta}\right) d y d \mu(s) \\
& =\frac{1}{\delta} \int_{-\delta}^{\delta} \int_{-K}^{K} \log |x-s-y| \psi\left(\frac{y}{\delta}\right) d \mu(s) d y
\end{aligned}
$$

Hence, for $\delta=\delta_{\varepsilon}$ and $x \in[-K, K],(4.49)-(4.51)$ imply

$$
\begin{aligned}
\left|\int_{-K-\delta_{\varepsilon}}^{K+\delta_{\varepsilon}} \log \right| x- & t\left|f_{\delta_{\varepsilon}}(t) d t-\Lambda(d \mu ; x)\right| \\
& \leqslant\left|\frac{1}{\delta_{\varepsilon}} \int_{-\delta_{\varepsilon}}^{\delta_{\varepsilon}} \psi\left(\frac{y}{\delta_{\varepsilon}}\right) \int_{-K}^{K}[\log |x-s-y|-\log |x-s|] d \mu(s) d y\right| \\
& \leqslant \frac{\varepsilon}{3}\left(\frac{1}{\delta_{\varepsilon}}\right) \int_{-\delta_{\varepsilon}}^{\delta_{\varepsilon}} \psi\left(\frac{y}{\delta_{\varepsilon}}\right) d y=\frac{\varepsilon}{3} .
\end{aligned}
$$

Finally, by the first part of the proof, there exists an integer $N$ and a sequence of polynomials $\left\{P_{n}(x)\right\}_{n=N}^{\infty}$ such that

$$
\frac{1}{n} \log \left|P_{n}(x)\right|<\frac{2 \varepsilon}{3}+\int_{-K-\delta_{\varepsilon}}^{K+\delta_{\varepsilon}} \log |x-t| f_{\delta_{\varepsilon}}(t) d t \quad \forall x \in[-K, K], n \geqslant N,
$$

and so, from (4.52), we get

$$
\frac{1}{n} \log \left|P_{n}(x)\right|<\varepsilon+\Lambda(d \mu ; x) \quad \forall x \in[-K, K], n \geqslant N .
$$

Proof of Theorem 2.9. In view of Lemma 4.3, the distribution $v(\alpha ; t) d t$ and its potential $\mathcal{L}(\alpha ; x)$ satisfy the hypotheses of Lemma 4.4. Combining (4.9) and (4.40), we see that for every $\varepsilon>0$, there exists an $N=N(\varepsilon)$ and sequence of monic polynomials $P_{n}(x)=x^{n}+\cdots \in \mathscr{P}_{n}, n=N, N+1, \ldots$, satisfying

$$
\frac{1}{n} \log \left|P_{n}(x)\right| \leqslant \varepsilon+\frac{|x|^{\alpha}}{\lambda_{\alpha}}-\log 2-\frac{1}{\alpha}, \quad \forall x \in[-1,1], n \geqslant N .
$$

With a change of variable, (4.53) can be written as

$$
\frac{1}{n} \log \left|\exp \left(-|x|^{\alpha}\right) Q_{n}(x)\right| \leqslant \varepsilon+\frac{1}{\alpha} \log \left(\frac{n}{\lambda_{\alpha}}\right)-\log 2-\frac{1}{\alpha},
$$

for all $x \in\left[-\left(n / \lambda_{\alpha}\right)^{1 / \alpha},\left(n / \lambda_{\alpha}\right)^{1 / \alpha}\right], n \geqslant N$, where

$$
Q_{n}(x)=x^{n}+\cdots \in \mathscr{P}_{n} \text {. }
$$

Hence, by (2.30) of Theorem 2.7, we have

$$
\left\|\exp \left(-|x|^{\alpha}\right) Q_{n}(x)\right\|_{(-\infty, \infty)}^{1 / n} \leqslant \frac{1}{2}\left(\frac{n}{\lambda_{\alpha} e}\right)^{1 / \alpha} e^{\varepsilon}, \quad n \geqslant N,
$$


and consequently

$$
\limsup _{n \rightarrow \infty} n^{-1 / \alpha}\left[E_{n}(\alpha)\right]^{1 / n} \leqslant \frac{1}{2}\left(\frac{1}{e \lambda_{\alpha}}\right)^{1 / \alpha} e^{\varepsilon} .
$$

Letting $\varepsilon \rightarrow 0$ in the last inequality and using Corollary 2.5, we obtain (2.38).

The proofs of Theorems 2.10 and 2.12 are postponed to $\$ 5$ where they follow from a more general argument (see the proofs of Theorems 3.6, 3.9 and 3.10).

5. Proofs of the results stated in $\$ 3$. In this section we adopt the same notation and conventions concerning constants as in $\S 3$. The proofs of Theorem 3.1 and Proposition 3.2 will be postponed to $\$ 6$ where they are derived from more general results.

Proof of TheOREM 3.3. In view of (3.10) and Theorem 3.1, we have for arbitrary $p, r$, with $0<p, r \leqslant \infty$,

$$
E_{n, p}^{1 / n} \leqslant\left\|w_{\alpha} T_{n, r}\right\|_{p}^{1 / n} \leqslant c_{1}^{1 / n} n^{c_{2} / n}\left\|w_{\alpha} T_{n, r}\right\|_{r}^{1 / n}=c_{1}^{1 / n} n^{c_{2} / n} E_{n, r}^{1 / n} .
$$

Together with Theorem 2.9, this implies (3.11) and then also (3.12).

Proof of TheOREM 3.4. As mentioned in $§ 3$, this is a straightforward consequence of Theorem 3.3 and Stirling's formula.

To prove Theorem 3.5 we require

Proposition. 5.1 [13, pp. 121, 127].

(a) Suppose $\left\{s_{n}\right\}_{n=1}^{\infty}$ is bounded $\left(C, k_{1}\right)$ and convergent $\left(C, k_{2}\right)$, where $k_{2}>k_{1}>-1$. Then, for each $k>k_{1}$, the sequence $\left\{s_{n}\right\}_{n=1}^{\infty}$ is convergent $(C, k)$.

(b) Suppose $\left\{s_{n}\right\}_{n=1}^{\infty}$ is convergent $(C, k)$ for some $k$, and there exists a constant $H$ such that

$$
n\left(s_{n+1}-s_{n}\right)>-H,
$$

for all $n$ large. Then $\left\{s_{n}\right\}_{n=1}^{\infty}$ is convergent.

Proof of Theorem 3.5. It is shown in [21] that, for each $\alpha \geqslant 1$,

$$
0<c_{1} \leqslant A_{n} \leqslant c_{2}, \quad n=1,2, \ldots,
$$

where $A_{n}$ is defined in (3.15). Hence the sequence $\left\{B_{n}\right\}_{n=1}^{\infty}$ is bounded $(C, 0)$ and, by Theorem 3.4, is convergent $(C, 1)$. Assertion (a) of Theorem 3.5 now follows from Proposition 5.1(a).

To prove part (b) of Theorem 3.5, write

$$
B_{n+1}-B_{n}=\log \left(\frac{A_{n+1}}{A_{n}}\right)=\log \left\{\frac{C_{n+1}}{C_{n}}\left(\frac{n}{n+1}\right)^{1 / \alpha}\right\} .
$$

The assumption (3.20) implies

$$
n\left(B_{n+1}-B_{n}\right) \geqslant \frac{n}{\alpha} \log \left(\frac{n}{n+1}\right)=-\frac{1}{\alpha} \log \left(1+\frac{1}{n}\right)^{n} \geqslant-\frac{1}{\alpha},
$$

for all $n$ large. Thus, by Proposition 5.1(b) and Theorem 3.4, the second part of Theorem 3.5 follows.

Proof of Theorem 3.6. The argument is similar to one used by Saff, Ullman, and Varga [28]. For the polynomials $Q_{n}$ of (3.22), we first show that

$$
\lim _{n \rightarrow \infty} \frac{1}{n} \log \left|Q_{n}(z)\right|=\mathcal{L}(\alpha ; z),
$$


uniformly for $z$ on every compact subset of $\mathbf{C}-[-K, K]$, where

$$
\mathcal{L}(\alpha ; z):=\int_{-1}^{1} \log z-t \mid v(\alpha ; t) d t .
$$

For this purpose, let $\varepsilon>0$ be given and note that, by (3.24), there exists an integer $n_{0}=n_{0}(\varepsilon)$ such that

$$
\left\|\exp \left(\frac{-n|x|^{\alpha}}{\lambda_{\alpha}}\right) Q_{n}(x)\right\|_{\infty,[-1,1]} \leqslant \frac{1}{2^{n}} \exp \left\{n\left(\varepsilon-\frac{1}{\alpha}\right)\right\} \quad \forall n \geqslant n_{0} .
$$

Now, Theorem 2.2 (with $a=\left(n / \lambda_{\alpha}\right)^{1 / \alpha}$ ) implies that, for all $z \in \mathbf{C}, n \geqslant n_{0}$,

$$
\exp \left(\frac{-n|z|^{\alpha}}{\lambda_{\alpha}}\right)\left|Q_{n}(z)\right| \leqslant \frac{1}{2^{n}} \exp \left\{n\left(\varepsilon-\frac{1}{\alpha}\right)\right\} G(\alpha ; z)^{n / \lambda_{\alpha}}|\phi(z)|^{n} .
$$

Thus, on taking the logarithm and recalling definition (2.9), we obtain

$$
u_{n}(z)-\mathcal{L}(\alpha ; z) \leqslant \varepsilon \quad \forall z \in \mathbf{C}, n \geqslant n_{0},
$$

where

$$
u_{n}(z):=\frac{1}{n} \log \left|Q_{n}(z)\right|
$$

Notice that, for each $n$, the function

$$
h_{n}(z):=u_{n}(z)-e(\alpha ; z)
$$

is harmonic in $\mathbf{C}^{*}-[-K, K]$, where $\mathbf{C}^{*}:=\mathbf{C} \cup\{\infty\}$. Furthermore, $h_{n}(\infty)=0$. From (5.10), it follows that the sequence $\left\{h_{n}\right\}$ forms a normal family in $\mathbf{C}^{*}-[-K, K]$. Let $h$ be an arbitrary limit function of the $h_{n}$. Then, by $(5.10)$ and the arbitrariness of $\varepsilon$, we have

$$
h(z) \leqslant 0 \quad \forall z \in \mathbf{C}^{*}-[-K, K] .
$$

But $h$ is harmonic and $h(\infty)=0$, so by the maximum principle, $h(z) \equiv 0$. Since this is true for every limit $h(z)$, we have $h_{n}(z) \rightarrow 0$ uniformly on every compact subset of $\mathrm{C}-[-K, K]$. This proves (5.6).

Notice that, for the measures $\nu_{n}$ of (3.23), we have

$$
\frac{1}{n} \frac{Q_{n}^{\prime}(z)}{Q_{n}(z)}=\int_{-K}^{K} \frac{d \nu_{n}(t)}{z-t} \quad \forall z \in \mathbf{C}-[-K, K],
$$

and consequently, from (5.6),

$$
\lim _{n \rightarrow \infty} \int_{-K}^{K} \frac{d \nu_{n}(t)}{z-t}=\int_{-1}^{1} \frac{v(\alpha ; t) d t}{z-t} \quad \forall z \in \mathbf{C}-[-K, K] .
$$

Since $\nu_{n}\{[-K, K]\}=1$ for all $n$, it follows from Helly's theorem (cf. [4]), that there exists a subsequence $\nu_{n_{i}}$ and a measure $\nu^{*}$ such that $\nu_{n_{i}} \rightarrow \nu^{*}$ weakly. For this subsequence,

$$
\lim _{i \rightarrow \infty} \int_{-K}^{K} \frac{d \nu_{n_{i}}(t)}{z-t}=\int_{-K}^{K} \frac{d \nu^{*}(t)}{z-t} \quad \forall z \in \mathbf{C}-[-K, K]
$$

and therefore, from (5.15),

$$
\int_{-K}^{K} \frac{d \nu^{*}(t)}{z-t}=\int_{-K}^{K} \frac{v(\alpha ; t) d t}{z-t} \quad \forall z \in \mathbf{C}-[-K, K],
$$


where $v(\alpha ; t)=0$ for $t \notin[-1,1]$. By the uniqueness of the Cauchy transform (cf. [12]), we have $\nu^{*}=\nu$, where $d \nu(t):=v(\alpha ; t) d t$. As this is true for every weak limit $\nu^{*}$, then $\nu_{n} \rightarrow \nu$ weakly. Equation (3.25) now follows from the fact that $\nu$ is absolutely continuous with respect to Lebesgue measure.

Proof of Proposition 3.8. Part (a) is an easy consequence of the extremal property (3.10) and the fact that if $z \in \mathbf{C}, z \notin \mathbf{R}$, then

$$
|x-z|^{r}>|x-\operatorname{Re}(z)|^{r} \quad \forall x \in \mathbf{R} .
$$

To prove part (b), let $\left\{t_{k, n}\right\}_{k=1}^{n}$ denote the (real) zeros of $T_{n, r}\left(\alpha ;\left(n / \lambda_{\alpha}\right)^{1 / \alpha} x\right)$ and, for fixed $\delta>0$, set

$$
\begin{gathered}
J_{1, n}:=\left\{k: t_{k, n}>1+\delta\right\}, \quad J_{2, n}:=\left\{k: t_{k, n}<-1-\delta\right\}, \\
m_{1, n}:=\left|J_{1, n}\right|, \quad m_{2, n}:=\left|J_{2, n}\right|, \quad m_{n}:=m_{1, n}+m_{2, n}, \\
q_{n}(x):=\prod_{k=1}^{n}\left(x-t_{k, n}\right)=\left(\frac{n}{\lambda_{\alpha}}\right)^{-n / \alpha} T_{n, r}\left(\alpha ;\left(n / \lambda_{\alpha}\right)^{1 / \alpha} x\right) .
\end{gathered}
$$

Let $\varepsilon>0$ be given and note that, from (3.12) of Theorem 3.3 (with $p=\infty$ ), we have, for each $n \geqslant n_{0}=n_{0}(\varepsilon)$,

$$
\frac{1}{2} \exp \left(\varepsilon-\frac{1}{\alpha}\right) \geqslant\left\|\exp \left(\frac{-n|x|^{\alpha}}{\lambda_{\alpha}}\right) q_{n}(x)\right\|_{\infty,[-1,1]}^{1 / n} .
$$

Put $t_{1}:=1+(\delta / 2), t_{2}:=-1-(\delta / 2)$ and define the monic polynomial

$$
\hat{q}_{n}(x):=q_{n}(x) \prod_{k \in J_{1, n}}\left(\frac{x-t_{1}}{x-t_{k, n}}\right) \cdot \prod_{k \in J_{2, n}}\left(\frac{x-t_{2}}{x-t_{k, n}}\right) .
$$

Notice that, for $x \in[-1,1]$,

$$
\prod_{k \in J_{1, n}}\left|\frac{x-t_{1}}{x-t_{k, n}}\right| \cdot \prod_{k \in J_{2, n}}\left|\frac{x-t_{2}}{x-t_{k, n}}\right| \leqslant\left(\frac{4+\delta}{4+2 \delta}\right)^{m_{1, n}}\left(\frac{4+\delta}{4+2 \delta}\right)^{m_{2, n}}=\left(\frac{4+\delta}{4+2 \delta}\right)^{m_{n}} \text {. }
$$

Thus, from (5.22) and (5.23),

$$
\left\|\exp \left(\frac{-n|x|^{\alpha}}{\lambda_{\alpha}}\right) q_{n}(x)\right\|_{\infty,[-1,1]}^{1 / n} \geqslant\left(1+\frac{\delta}{4+\delta}\right)^{m_{n} / n}\left\|\exp \left(\frac{-n|x|^{\alpha}}{\lambda_{\alpha}}\right) \hat{q}_{n}(x)\right\|_{\infty,[-1,1]}^{1 / n} .
$$

Next, from (2.30) of Theorem 2.7 and Corollary 2.5, we have

$$
\left\|\exp \left(\frac{-n|x|^{\alpha}}{\lambda_{\alpha}}\right) \hat{q}_{n}(x)\right\|_{\infty,[-1,1]}^{1 / n} \geqslant\left(\frac{n}{\lambda_{\alpha}}\right)^{-1 / \alpha}\left[E_{n}(\alpha)\right]^{1 / n} \geqslant \frac{1}{2} \exp \left(-\frac{1}{\alpha}\right) .
$$

Hence, by (5.21), (5.24) and (5.25),

$$
\frac{1}{2} \exp \left(\varepsilon-\frac{1}{\alpha}\right) \geqslant\left(1+\frac{\delta}{4+\delta}\right)^{m_{n} / n} \frac{1}{2} \exp \left(-\frac{1}{\alpha}\right), \quad n \geqslant n_{0} .
$$

As $\varepsilon>0$ is arbitrary, the last inequality implies $m_{n} / n \rightarrow 0$ as $n \rightarrow \infty$.

Proof of Theorem 3.9. In the proof of Proposition 3.8(b), let $\delta=1$ so that the polynomials $\hat{q}_{n}(x)$ defined in (5.22) have all their zeros in $[-2,2]$. Since $m_{n} / n \rightarrow 0$ 
as $n \rightarrow \infty$, we see from (5.21) and (5.24) that the $\hat{q}_{n}(x)$ satisfy the hypothesis (3.24) of Theorem 3.6. Thus (3.31) is a consequence of (3.25) and Proposition 3.8(b).

Proof OF TheOREM 3.10. Continuing with the polynomials $\hat{q}_{n}$ of the preceding proof, it follows from (5.6) that

$$
\lim _{n \rightarrow \infty}\left|\hat{q}_{n}(z)\right|^{1 / n}=\exp \left(\int_{-1}^{1} \log |z-t| v(\alpha ; t) d t\right),
$$

uniformly on every compact subset of $\mathbf{C}-[-2,2]$. By Proposition 3.8, equation (5.26) holds with $\hat{q}_{n}$ replaced by $q_{n}$ (cf. (5.20)) provided $z \notin \mathbf{R}$. This proves equation (3.32). When $r=\infty$, all the zeros of $T_{n, r}\left(\alpha ;\left(n / \lambda_{\alpha}\right)^{1 / \alpha} x\right)$ lie in $[-1,1]$ and the uniform convergence in (3.32) is valid on compact subsets of $\mathbf{C}-[-1,1]$. For $r=2$, the last assertion of Theorem 3.10 likewise follows from the remarks preceding (3.27).

6. Weighted analogues of Nikolskii-type inequalities. In this section, we derive inequalities relating different weighted $L^{r}$-metrics of algebraic polynomials not exceeding a fixed degree. Since the class of all such polynomials is a finite-dimensional vector space, the metrics are all equivalent. The objectives of these inequalities is to estimate the constants involved in this equivalence in terms of the degree of the polynomials in question. Similar inequalities for trigonometric polynomials and entire functions of finite exponential type were obtained by S. M. Nikolskii (cf. [31, $\S 4.9 .2 ; 25, \S 3.3])$. Inequalities relating weighted $L^{r}$-norms of algebraic polynomials were obtained by Mhaskar [19] in connection with his investigations on the smoothness of Fourier transforms. In this section, we shall extend these inequalities to all $L^{r}$-metrics, $r>0$, give simpler proofs and sharper constants.

For suitable weight functions $w$ on $\mathbf{R}$, we let

$$
\left\{p_{n}\left(w^{2} ; x\right)=\gamma_{n} x^{n}+\cdots \in \mathscr{P}_{n}, \gamma_{n}>0\right\}
$$

be the sequence of polynomials orthonormal with respect to $w^{2}$ on $\mathbf{R}$, i.e.

$$
\int_{-\infty}^{\infty} p_{n}\left(w^{2} ; x\right) p_{m}\left(w^{2} ; x\right) w^{2}(x) d x=\delta_{n, m} .
$$

For each $n=0,1, \ldots$, define

$$
\begin{gathered}
K_{n}(w ; x, t):=\sum_{j=0}^{n} p_{j}\left(w^{2} ; x\right) p_{j}\left(w^{2} ; t\right), \\
M_{n}(w):=\left\|w^{2}(x) K_{n}(w ; x, x)\right\|_{\infty},
\end{gathered}
$$

where the sup norm in (6.3) is taken over $\mathbf{R}$. We shall write $p_{n}(x)$ instead of $p_{n}\left(w^{2} ; x\right)$ when the weight function is clear from the context; the same convention applies to other quantities related to $w$.

THEOREM 6.1. Suppose that $\|w\|_{2}>0$ and $x^{n} w(x) \in L^{q}(\mathbf{R}), n=0,1, \ldots$, for every $q$ with $0<q \leqslant \infty$. Let $0<p<r \leqslant \infty$ and $\pi_{n} \in \mathscr{P}_{n}$. Then

$$
\left\|w \pi_{n}\right\|_{r} \leqslant\left\{M_{k n}\left(w^{k}\right)\right\}^{1 / p-1 / r}\left\|w \pi_{n}\right\|_{p},
$$

where $k$ is any integer such that $2 k \geqslant p$. 
Proof. Fix an integer $k$ with $2 k \geqslant p$. There are constants $b_{0}, b_{1}, \ldots, b_{k n}$ such that the polynomial $\left[\pi_{n}(x)\right]^{k} \in \mathscr{P}_{n k}$ can be expressed as

$$
\left[\pi_{n}(x)\right]^{k}=\sum_{j=0}^{k n} b_{j} p_{j}\left(w^{2 k} ; x\right)
$$

and

$$
\sum_{j=0}^{k n} b_{j}^{2}=\int_{-\infty}^{\infty}\left[\pi_{n}(x) w(x)\right]^{2 k} d x .
$$

Applying the Cauchy-Schwarz inequality in (6.5) and using (6.6), we get

$$
\left\|w \pi_{n}\right\|_{\infty}^{2 k} \leqslant\left\|\sum_{j=0}^{k n} p_{j}^{2}\left(w^{2 k} ; x\right) w^{2 k}(x)\right\|_{\infty}\left\|w \pi_{n}\right\|_{2 k}^{2 k} .
$$

Thus

$$
\left\|w \pi_{n}\right\|_{\infty} \leqslant L_{n}^{1 / 2 k}\left\|w \pi_{n}\right\|_{2 k}
$$

where

$$
L_{n}:=M_{k n}\left(w^{k}\right)
$$

Now,

$$
\begin{aligned}
\left\|w \pi_{n}\right\|_{2 k}^{2 k} & =\int_{-\infty}^{\infty}\left|w(x) \pi_{n}(x)\right|^{2 k-p}\left|w(x) \pi_{n}(x)\right|^{p} d x \\
& \leqslant\left\|w \pi_{n}\right\|_{\infty}^{2 k-p}\left\|w \pi_{n}\right\|_{p}^{p} \leqslant L_{n}^{1-p / 2 k}\left\|w \pi_{n}\right\|_{2 k}^{2 k-p}\left\|w \pi_{n}\right\|_{p}^{p},
\end{aligned}
$$

where the last inequality follows from (6.8). Hence,

$$
\left\|w \pi_{n}\right\|_{2 k} \leqslant L_{n}^{1 / p-1 / 2 k}\left\|w \pi_{n}\right\|_{p} .
$$

Using (6.10) in (6.8), we see that

$$
\left\|w \pi_{n}\right\|_{\infty} \leqslant L_{n}^{1 / p}\left\|w \pi_{n}\right\|_{p}
$$

which establishes (6.4) for the case $r=\infty$. Now, let $0<r<\infty$. Then on using

$$
\begin{aligned}
\left\|w \pi_{n}\right\|_{r}^{r} & =\int_{-\infty}^{\infty}\left|w(x) \pi_{n}(x)\right|^{r-p}\left|w(x) \pi_{n}(x)\right|^{p} d x \\
& \leqslant\left\|w \pi_{n}\right\|_{\infty}^{r-p}\left\|w \pi_{n}\right\|_{p}^{p} \leqslant L_{n}^{r / p-1}\left\|w \pi_{n}\right\|_{p}^{r-p}\left\|w \pi_{n}\right\|_{p}^{p},
\end{aligned}
$$

which yields (6.4).

In the case when the weight function $w$ is supported on a finite interval and $0<p<r \leqslant \infty$, Hölder's inequality can be used to obtain an upper bound for the $L^{p}$-norm in terms of the $L^{r}$-norm. For an arbitrary weight function supported on an infinite interval, such an estimation seems to be much harder. In what follows we shall show that, under certain technical conditions on the weight function, we can reduce this problem to the simpler one of estimating the norms on finite intervals. All the conditions which we impose will be satisfied, in particular, by $\exp \left(-|x|^{\alpha}\right)$, $\alpha>0$. 
Definition 6.2. We say that a weight function $w$ is in the class $\Xi$ if the following three conditions hold:

(i) $\|w\|_{2}>0$ and $x^{n} w(x) \in L^{q}(\mathbf{R})$ for all $0<q \leqslant \infty, n=0,1, \ldots$;

(ii) for each $k=1,2, \ldots$,

$$
\limsup _{n \rightarrow \infty}\left\{M_{k n}\left(w^{k}\right)\right\}^{1 / n}=: \mu(w, k)=\mu<\infty ;
$$

(iii) there exists a nondecreasing sequence $\left\{\zeta_{n}\right\}_{n=1}^{\infty}$ of positive numbers such that

$$
\left\|w P_{n}\right\|_{\infty,\left[-\zeta_{n}, \zeta_{n}\right]}=\left\|w P_{n}\right\|_{\infty, \mathbf{R}} \quad \forall P_{n} \in \mathscr{P}_{n}, n=1,2, \ldots,
$$

and

$$
\limsup _{n \rightarrow \infty} \zeta_{n}^{1 / n}=: B(w)=B<\infty .
$$

LEMMA 6.3. Suppose $w \in \Xi$ and $0<p<\infty$. Then there exist positive constants $c_{1}$, $c_{2}, A$, depending only on $p$ and $w$, such that for every $P_{n} \in \mathscr{P}_{n}$,

$$
\left\|w P_{n}\right\|_{p}^{p} \leqslant\left(1+c_{1} e^{-c_{2} n}\right) \int_{|x| \leqslant A \zeta_{2 n+1}}\left|w(x) P_{n}(x)\right|^{p} d x, \quad n=1,2, \ldots
$$

where $l:=1+[1 / p]$ and $\left\{\zeta_{n}\right\}$ satisfies condition (iii) of Definition 6.2.

PROof. For $P_{n} \in \mathscr{P}_{n}, n \geqslant 1$, and $l=1+[1 / p]$, property (6.14) implies that

$$
\left|x^{n+l} P_{n}(x) w(x)\right| \leqslant \zeta_{2 n+l}^{n+l}\left\|w P_{n}\right\|_{\infty} \text { a.e. on } \mathbf{R},
$$

and hence

$$
\left|w(x) P_{n}(x)\right| \leqslant\left(\frac{\zeta_{2 n+l}^{1+(l / n)}}{|x|}\right)^{n} \frac{1}{|x|^{l}}\left\|w P_{n}\right\|_{\infty} \quad \text { a.e. on } \mathbf{R} .
$$

From (6.15) we see that there is a positive constant $c_{3}=c_{3}(w, p)$ such that

$$
\left|w(x) P_{n}(x)\right| \leqslant\left(\frac{c_{3} \zeta_{2 n+l}}{|x|}\right)^{n} \frac{1}{|x|^{l}}\left\|w P_{n}\right\|_{\infty} \text { a.e. on } \mathbf{R} \text {. }
$$

We can estimate $\left\|w P_{n}\right\|_{\infty}$ from above in terms of $\left\|w P_{n}\right\|_{p}$ by using Theorem 6.1 with $k=1+[p / 2]$. Then, in view of (6.13), there exists a positive constant $c_{4}=c_{4}(w, p)$ such that

$$
\left|w(x) P_{n}(x)\right| \leqslant\left(\frac{c_{4} \zeta_{2 n+l}}{|x|}\right)^{n} \frac{1}{|x|^{l}}\left\|w P_{n}\right\|_{p} \quad \text { a.e. on } \mathbf{R} \text {. }
$$

Hence, with

$$
A:=\max \left\{2 c_{4}, \frac{1}{\zeta_{1}}\left(\frac{2}{l p-1}\right)^{1 /(l p-1)}\right\},
$$

we have from (6.20) and the fact that $l p>1$

$$
\begin{aligned}
\int_{|x|>A \zeta_{2 n+l}}\left|w(x) P_{n}(x)\right|^{p} d x & \leqslant 2^{-n p}\left\|w P_{n}\right\|_{p}^{p} \int_{|x|>A \zeta_{2 n+l}}|x|^{-l p} d x \\
& \leqslant 2^{-n p}\left\|w P_{n}\right\|_{p}^{p} \int_{|x|>A \zeta_{1}}|x|^{-l p} d x \leqslant 2^{-n p}\left\|w P_{n}\right\|_{p}^{p} .
\end{aligned}
$$


Finally, on writing

$$
\left\|w P_{n}\right\|_{p}^{p}=\int_{|x| \leqslant A \zeta_{2 n+l}}\left|w(x) P_{n}(x)\right|^{p} d x+\int_{|x|>A \zeta_{2 n+l}}\left|w(x) P_{n}(x)\right|^{p} d x
$$

and using (6.21), the inequality (6.16) follows.

An application of the Hölder inequality and Lemma 6.3 gives the following.

TheOREM 6.4. Suppose $w \in \Xi, 0<p<r \leqslant \infty$, and $P_{n} \in \mathscr{P}_{n}$. Then there exists a constant $c_{1}$ depending only on $w, p, r$ such that

$$
\left\|w P_{n}\right\|_{p} \leqslant c_{1} \zeta_{2 n+l}^{(1 / p-1 / r)}\left\|w P_{n}\right\|_{r}
$$

where $\left\{\zeta_{n}\right\}$ satisfies condition (iii) of Definition 6.2 and $l$ is any integer such that $l \geqslant 1+[1 / p]$.

Our next task is to show that the weights $w_{\alpha}(x)=\exp \left(-|x|^{\alpha}\right)$ are in class $\Xi$ if $\alpha>0$. It is obvious that $x^{n} w_{\alpha}(x) \in L^{q}(\mathbf{R})$ for every $0<q \leqslant \infty$, so that condition (i) of Definition 6.2 is satisfied. By (2.30) of Theorem 2.7, the condition (iii) is also satisfied by $w_{\alpha}$; namely, we can choose $\zeta_{n}=a_{n}(\alpha)$ which is a constant multiple of $n^{1 / \alpha}$. Thus it remains to estimate the Christoffel functions in order to verify the condition (ii). Because of the homogeneity

$$
M_{k n}\left(w_{\alpha}^{k}\right)=k^{1 / \alpha} M_{k n}\left(w_{\alpha}\right),
$$

it suffices to estimate $M_{n}\left(w_{\alpha}\right)$. Such estimates were obtained by Freud for a very general class of weight functions. The class for which his results are published [8] includes $w_{\alpha}$ if $\alpha \geqslant 2$. Freud generalized these results to include $w_{\alpha}$ for $\alpha>1$ during a course taught at Ohio State University. The case of the weight $w_{1}$ appears in [11]. Using the preceding results of this section and the known result for $w_{1}$, we can give a simple generalization of these estimates which applies to the class of all weights $w_{\alpha}$, $\alpha>0$. This is done in

THEOREM 6.5. Let $w_{\alpha}(x)=\exp \left(-|x|^{\alpha}\right), \alpha>0$.

(a) If $0<\alpha<1$, then

$$
M_{n}\left(w_{\alpha}\right) \leqslant c_{1} n, \quad n=1,2, \ldots
$$

(b) If $\alpha \geqslant 1$, then

$$
M_{n}\left(w_{\alpha}\right) \leqslant c_{1} n^{1-1 / \alpha} \log n, \quad n=2,3, \ldots
$$

(c) If $\alpha \geqslant 2$, then (6.25) can be replaced by

$$
M_{n}\left(w_{\alpha}\right) \leqslant c_{1} n^{1-1 / \alpha}, \quad n=1,2, \ldots
$$

Here $c_{1}$ is a constant depending only on $\alpha$.

To prove Theorem 6.5 we first recall the following estimate which appears in [11].

LEMMA 6.6. For every $x \in \mathbf{R}$ and $n=2,3, \ldots$,

$$
w_{1}(x) \sum_{j=0}^{n} p_{j}^{2}\left(w_{1}^{2} ; x\right) \leqslant c_{1} \log \left(\frac{n+e|x|}{1+|x|}\right) \text {. }
$$


REMARKS. (i) In the previous sections, $p_{j}\left(w_{1}^{2} ; x\right)$ was denoted by $p_{j}(1 ; x)$.

(ii) In [11, Theorem 2.1], the estimate (6.27) is formally stated only for $|x| \leqslant c_{2} n$. However, it is remarked in [11] that (6.27) holds for all $x \in \mathbf{R}$. This extension from the finite interval $|x| \leqslant c_{2} n$ to the whole real line is also an easy consequence of (2.31) (cf. [8]).

Since the right-hand side of (6.27) is a decreasing function of $|x|$, we deduce

COROLlARY 6.7. For $n=2,3, \ldots$,

$$
M_{n}\left(w_{1}\right) \leqslant c_{1} \log n .
$$

We can now give the

Proof OF TheOREM 6.5. (a) Let $0<\alpha<1$. Since the function $t^{\alpha}-(t-x)^{\alpha}$ is decreasing in $t$ if $t>x>0$, we see that

$$
|t|^{\alpha} \leqslant|x|^{\alpha}+|t-x|^{\alpha} \quad \forall t, x \in \mathbf{R} .
$$

Now, for $x \in \mathbf{R}$ (cf. [4, §I.4]),

$$
\begin{aligned}
\left(\sum_{j=0}^{n} p_{j}^{2}\left(w_{\alpha}^{2} ; x\right)\right)^{-1} & =\inf _{\pi_{n} \in \mathscr{P}_{n}} \pi_{n}^{-2}(x) \int_{-\infty}^{\infty} \pi_{n}^{2}(t) \exp \left(-2|t|^{\alpha}\right) d t \\
& \geqslant \exp \left(-2|x|^{\alpha}\right) \inf _{\pi_{n} \in \mathscr{P}_{n}} \pi_{n}^{-2}(x) \int_{-\infty}^{\infty} \pi_{n}^{2}(t) \exp \left(-2|t-x|^{\alpha}\right) d t \\
& =\frac{1}{2^{1 / \alpha}} w_{\alpha}^{2}(x) \inf _{\pi_{n} \in \mathscr{P}_{n}} \pi_{n}^{-2}(0) \int_{-\infty}^{\infty} \pi_{n}^{2}(t) \exp \left(-2|t|^{\alpha}\right) d t \\
& \geqslant \frac{e^{-2}}{2^{1 / \alpha}} w_{\alpha}^{2}(x) \inf _{\pi_{n} \in \mathscr{P}_{n}} \pi_{n}^{-2}(0) \int_{-1}^{1} \pi_{n}^{2}(t) d t \\
& \geqslant \frac{c_{1} w_{\alpha}^{2}(x)}{n}
\end{aligned}
$$

the last inequality follows from well-known representations for the constant terms of normalized Legendre polynomials (cf. [30, §4.7]). This proves (6.24).

(b) If $\alpha=1$, then (6.25) coincides with (6.28). Thus we need to consider only the case when $\alpha>1$. In view of (6.28), we have $w_{1} \in \Xi$. Hence, by Lemma 6.3 (with $\zeta_{n}=\pi n / 2, p=2$ ), there is a constant $D_{1}>1$ such that if $P_{n} \in \mathscr{P}_{n}$ and $n$ is sufficiently large, then

$$
\int_{|x| \leqslant D_{1} n}\left|w_{1}(x) P_{n}(x)\right|^{2} d x \geqslant \frac{1}{4} \int_{-\infty}^{\infty}\left|w_{1}(x) P_{n}(x)\right|^{2} d x .
$$

By (2.30) of Theorem 2.7, we may also assume $D_{1}$ to be so large that, for every $P_{2 n} \in \mathscr{P}_{2 n}$,

$$
\max _{|x| \leqslant D_{1} n^{1 / \alpha}}\left|w_{\alpha}^{2}(x) P_{2 n}(x)\right|=\left\|w_{\alpha}^{2}(x) P_{2 n}(x)\right\|_{\infty} .
$$

Note that if $|t|,|x| \leqslant 2 D_{1} n^{1 / \alpha}$, then since $\alpha>1$ we have

$$
|t|^{\alpha} \leqslant|x|^{\alpha}+\alpha D_{2} n^{1-1 / \alpha}|t-x|, \quad D_{2}:=\left(2 D_{1}\right)^{\alpha-1} .
$$


Therefore, if $|x| \leqslant D_{1} n^{1 / \alpha}$,

$$
\begin{aligned}
\left(\sum_{j=0}^{n} p_{j}^{2}\left(w_{\alpha}^{2} ; x\right)\right)^{-1}=\inf _{\pi_{n} \in \mathscr{T}_{n}} \pi_{n}^{-2}(x) \int_{-\infty}^{\infty} \pi_{n}^{2}(t) w_{\alpha}^{2}(t) d t \\
\quad \geqslant \inf _{\pi_{n} \in \mathscr{P}_{n}} \pi_{n}^{-2}(x) \int_{|t-x| \leqslant D_{1} n^{1 / \alpha}} \pi_{n}^{2}(t) \exp \left(-2|t|^{\alpha}\right) d t \\
\quad \geqslant \inf _{\pi_{n} \in \mathscr{P}_{n}} \pi_{n}^{-2}(x) \int_{|t-x| \leqslant D_{1} n^{1 / \alpha}} \pi_{n}^{2}(t) \exp \left(-2|x|^{\alpha}-2 \alpha D_{2} n^{1-1 / \alpha}|t-x|\right) d t \\
=\frac{c}{n^{1-1 / \alpha}} w_{\alpha}^{2}(x) \inf _{\pi_{n} \in \mathscr{P}_{n}} \pi_{n}^{-2}(0) \int_{|u| \leqslant \alpha 2^{\alpha-1} D_{1}^{\alpha} n} \pi_{n}^{2}(u) w_{1}^{2}(u) d u,
\end{aligned}
$$

where $c$ is a positive constant. Since $D_{1}>1$ and $\alpha>1$, the inequalities (6.34), (6.31) and (6.27) imply that, for all $n$ large,

$$
\begin{aligned}
\left(\sum_{j=0}^{n} p_{j}^{2}\left(w_{\alpha}^{2} ; x\right)\right)^{-1} & \geqslant \frac{c}{4 n^{1-1 / \alpha}} w_{\alpha}^{2}(x) \inf _{\pi_{n} \in \mathscr{P}_{n}} \pi_{n}^{-2}(0) \int_{-\infty}^{\infty} \pi_{n}^{2}(u) w_{1}^{2}(u) d u \\
& \geqslant \frac{c_{1} w_{\alpha}^{2}(x)}{n^{1-1 / \alpha} \log n}, \quad \text { if }|x| \leqslant D_{1} n^{1 / \alpha}
\end{aligned}
$$

The desired inequality (6.25) now follows from (6.35) and (6.32).

(c) See [8].

We are now able to prove Theorem 3.1 and Proposition 3.2.

Proof of Theorem 3.1. Inequality (3.2) is a consequence of Theorem 6.1, the estimates for the Christoffel functions given in Theorem 6.5, and (6.23). Since $w_{\alpha} \in \Xi, \alpha>0$, with $\zeta_{n}=a_{n}(\alpha)=\left(n / \lambda_{\alpha}\right)^{1 / \alpha}$, inequality (3.6) follows from Theorem 6.4 .

Proof of Proposition 3.2. Inequality (3.8) is an immediate consequence of Lemma 6.3, where again we use the fact that $w_{\alpha} \in \Xi, \alpha>0$, with $\zeta_{n}=a_{n}(\alpha)$.

ADDED IN PROOF. The authors have recently become aware of the independent work of E. A. Rakhmanov [Math. USSR Sb. 47 (1984), 155-193] which deals with the asymptotic properties of the polynomials $p_{n}(\alpha ; x)$ orthonormal with respect to the weight $\exp \left(-2|x|^{\alpha}\right)$. Rakhmanov considers only $\alpha>1$, but he proves that $\left(n / \lambda_{\alpha}\right)^{-1 / \alpha} X_{n}(\alpha) \rightarrow 1$ as $n \rightarrow \infty$, where $X_{n}(\alpha)$ is the largest zero of $p_{n}(\alpha ; x)$. Apparently, Rakhmanov, in turn, was unaware of the earlier works of Freud, Nevai and Ullman. Concerning Freud's conjecture, Al. Magnus has recently shown that it is true for $\alpha$ an even integer.

\section{REFERENCES}

1. L. V. Ahlfors, Complex analysis (2nd ed.), McGraw-Hill, New York, 1966.

2. A. Erdélyi, W. Magnus, F. Oberhettinger and F. G. Tricomi, Higher transcendental functions, Vol. I, McGraw-Hill, New York, 1953.

3. P. Erdös and P. Turan, On the uniformly dense distribution of certain sequences of points, Ann. of Math. 41 (1940), 162-173.

4. G. Freud, Orthogonal polynomials, Pergamon Press, London, 1971.

5. __ On two polynomial inequalities. I, Acta Math. Acad. Sci. Hungar. 22 (1971), 109-116.

6. On the greatest zero of an orthogonal polynomial. II, Acta Sci. Math. (Szeged) 36 (1974), 45-54. 
7. On estimations of the greatest zeroes of orthogonal polynomials, Acta Math. Acad. Sci. Hungar. 25 (1974), 99-107.

8. On polynomial approximation with respect to general weights, Lecture Notes in Math., vol. 399, Springer-Verlag, Berlin and New York, 1974, pp. 149-179.

9. Acad. Sect. A 76 (1976), 1-6.

10. On the greatest zero of an orthogonal polynomial (preprint), Abstract 753-B36, Notices Amer. Math. Soc. 25 (1978).

11. G. Freud, A. Giroux, and Q. I. Rahman, Sur l'approximation polynomiale avec poids $\exp (-|x|)$, Canad. J. Math. 30 (1978), 358-372.

12. John Garnett, Analytic capacity and measure, Lecture Notes in Math., vol. 297, Springer-Verlag, Berlin and New York, 1972.

13. G. H. Hardy, Divergent series, Oxford Univ. Press, Oxford, 1949.

14. N. S. Landkof, Foundations of modern potential theory, Springer-Verlag, Berlin and New York, 1972.

15. G. G. Lorentz, Approximation by incomplete polynomials (problems and results), Padé and Rational Approximations: Theory and Applications, edited by E. B. Saff and R. S. Varga, Academic Press, New York, 1977, pp. 289-302.

16. A. I. Markushevich, Theory of functions of a complex variable, Volume II, Prentice-Hall, Englewood Cliffs, N. J., 1967.

17. H. N. Mhaskar, Weighted polynomial approximation of entire functions. I, J. Approx. Theory 35 (1982), 203-213.

18. W W W W W _ Whted polynomial approximation of entire functions. II, J. Approx. Theory 33 (1981), 59-68.

19. Weighted analogues of Nikolskii-type inequalities and their applications, Conf. Harmonic

Anal. in honor of A. Zygmund (edited by Beckner, Calderon, Fefferman, Jones), Vol. II, Wadsworth International, Belmont, 1983, pp. 783-801.

20. On the convergence of expansions in polynomials orthogonal with respect to general weight functions on the whole real line, Acta Math. Acad. Sci. Hungar. (to appear).

21. P. Nevai, Polynomials orthogonal on the real line with respect to $|x|^{\alpha} \exp \left(-|x|^{\beta}\right)$. I, Acta Math. Acad. Sci. Hungar. 24 (1973), 335-342. (Russian)

22. __ Orthogonal polynomials, Mem. Amer. Math. Soc. No. 213 (1979).

23. - Orthogonal polynomials associated with $\exp \left(-x^{4}\right)$, CMS Conference Proceedings, Vol. 3 (Second Edmonton Conference on Approximation Theory), Amer. Math. Soc., Providence, R.I., 1982, pp. 263-285.

24. S. M. Nikolskii, Inequalities for integral functions of finite degree and their applications in the theory of different functions of many variables, Trudy Mat. Inst. Steklov. 38 (1951), 244-278.

25. Approximation of functions of several variables and imbedding theorems, Springer-Verlag, Berlin and New York, 1975.

26. G. Pòlya and G. Szegö, Über den transfiniten Durchmesser (Kapäzitatskonstante) von ebenen und räumlichen Punktmengen, J. Reine Angew. Math. 165 (1931), 4-49.

27. H. L. Royden, Real analysis (2nd ed.), Macmillan, New York, 1968.

28. E. B. Saff, J. L. Ullman, and R. S. Varga, Incomplete polynomials: an electrostatics approach, Approximation Theory. III, edited by E. W. Cheney, Academic Press, New York, 1980, pp. 769-782.

29. E. B. Saff and R. S. Varga, On incomplete polynomials. II, Pacific J. Math. 92 (1981), 161-172.

30. G. Szegö, Orthogonal polynomials (3rd ed.), Amer. Math. Soc. Colloq. Publ., vol. 23, Amer. Math. Soc., Providence, R.I., 1967.

31. A. F. Timan, Theory of approximation of functions of a real variable, Macmillan, New York, 1963.

32. J. L. Ullman, On the regular behavior of orthogonal polynomials, Proc. London Math. Soc. (3) 24 (1972), 119-148.

33. __ Orthogonal polynomials associated with an infinite interval, Michigan Math. J. 27 (1980), $353-363$.

34. On orthogonal polynomials associated with the infinite interval, Approximation Theory. III, edited by E. W. Cheney, Academic Press, New York, 1980, pp. 889-895.

Department of Mathematics, California State University, los Angeles, California 90032.

Center for Mathematical Services, University of South Florida, Tampa, Florida 33620 